\title{
论 文
}

\section{云南寒武纪早期澄江动物群古群落分析}

\author{
赵方臣 ${ }^{(1)}$, 朱茂炎 ${ }^{(1)}$, 胡世学 ${ }^{(1)}$ \\ (1) 中国科学院南京地质古生物研究所, 现代古生物学和地层学国家重点实验室, 南京 210008; \\ (2) 中国地质调查局成都地质调查中心, 成都 610081 \\ * 联系人, E-mail: myzhu@nigpas.ac.cn
}

收稿日期: 2010-05-06; 接受日期: 2010-08-20

国家自然科学基金(批准号: 40725005, 40930211, J0630967)、国家重点基础研究发展计划项目(编号: 2006CB806401)资助

摘要 本研究将澄江动物群分属于 18 个动物门和类群的 228 个化石物种区分为 23 个生态 功能群, 并依据澄江-海口-安宁地区野外系统采集的化石个体丰度数据(114 个物种, 18406 个 体), 对寒武纪早期澄江动物群总体分异度、物种组成和结构以及生态类型进行了定量分析. 结果显示节肢动物无论是物种丰度 $(37 \%)$ 还是个体丰度 $(51.8 \%)$ 都最为丰富, 是动物群中的优 势类群; 曳鳃动物 $(22.6 \%)$ 和腕足动物 (16.3\%) 的个体丰度也相对较高. 其中, 节肢动物的 Kunmingella douvillei(26.2\%)、曳鳃动物的 Cricocosmia jinningensis $(15.4 \%)$ 、腕足动物的 Diandongia pista $(11 \%)$ 是个体丰度最高三个物种, 占整个动物群个体数量的 $52.6 \%$. 澄江动物 群不同生活方式的各类生物定量分析表明，生物占据了海洋水体各空间领域，从底内生物到 浮游生物都有化石代表. 其中, 以表栖动物为主 (物种数 $=63 \%$, 个体数 $=68.4 \%$ ), 其次是底内 动物 (物种数 $=11.9 \%$, 个体数 $=25.9 \%$ ). 游泳动物 (物种数 $=11.5 \%$, 个体数 $=2.6 \%$ ) 和浮游动物 (物 种数 $=5.3 \%$, 个体数 $=3.1 \%$ ) 化石保存较少. 澄江动物群中动物的取食方式主要以滤食( 物种 数 $=35.6 \%$, 个体数 $=26.1 \%$ ) 和捕食 $/$ 食腐 (物种数 $=31.1 \%$, 个体数 $=40.4 \%$ ) 为主. 物种丰度的最高 生态功能群是表栖固着滤食类 $(18.5 \%)$ 、表栖活动捕食或食腐类 $(16.3 \%)$ 、表栖活动滤食类 (7.9\%)和近底游泳滤食类 $(6.6 \%)$, 而个体丰度最高的生态功能群是表栖活动杂食类 $(28.2 \%)$ 、内 栖活动捕食或食腐类 $(19.8 \%)$ 、表栖固着滤食类 $(17.7 \%)$ 和表栖活动捕食或食腐类 $(15.3 \%)$. 以上 分析结果表明澄江动物群由丰富多样的门类和大量具有不同复杂生态功能的物种组成, 证明 了寒武纪早期物种间高强度捕食压力的存在, 并形成了由多层次营养级结构构成的金字塔式 复杂食物网, 为寒武纪早期的物种多样性和复杂生态系统提供具体科学依据.
关键词

澄江动物群 寒武纪早期 帽天山 群落生态学
发现于我国云南的澄江动物群是全球寒武纪最 早的特异埋藏化石群(图 1(a)), 保存了大量具软躯体 或弱矿化外壳的后生动物化石, 展示了寒武纪大辐 射时期海洋生态系统和后生动物多样性特征, 是全
球研究寒武纪大爆发演化事件最重要的化石宝库之 —[2 6]. 自发现以来的 20 多年里 ${ }^{[7]}$, 澄江动物群的系 统古生物学、功能形态学和个体生态学研究取得了极 为显著的学术成就, 发表的物种数量超过 200 种. 然

引用格式: Zhao F C, Zhu M Y, Hu S X. Community structure and composition of the Cambrian Chengiang biota. Sci China Earth Sci, 2010, doi: $10.1007 / \mathrm{s} 11430-010-4087-8$ 


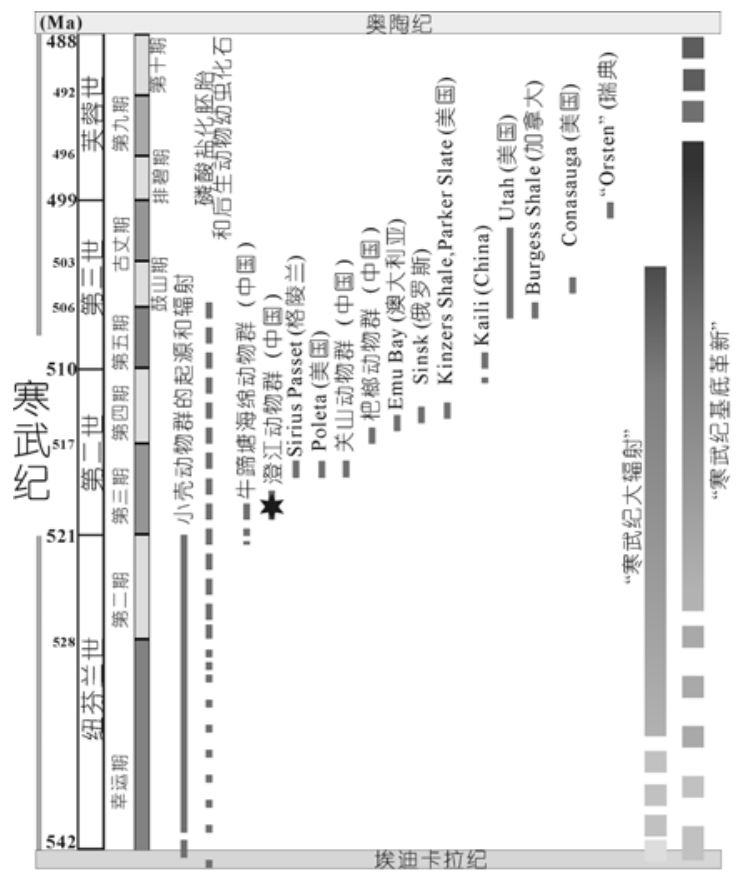

(a)

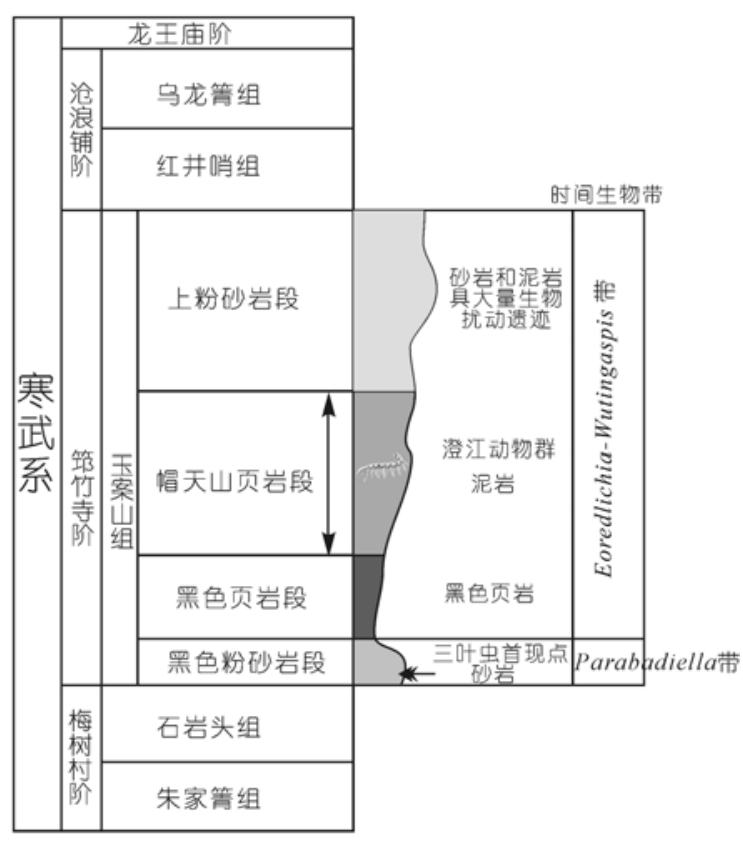

(b)

图 1 澄江动物群地质年代和地层

(a) 寒武纪特异埋藏化石库的时间分布(据Zhu等 ${ }^{[1]}$ 修改); (b) 滇东南澄江地区澄江动物群产出层位和生物带

而, 有关澄江动物群系统全面的定量古生态学研究 一直以来没有受到重视. 为了揭示寒武纪大爆发过 程中动物多样性和生态系统演化, 本文依据已发表 文献物种数据和采用新的化石采集方法获得的个体 丰度数据, 对澄江动物群总体分异度、群落组成和结 构以及生态类型进行了定量分析.

\section{1 化石分布、地质背景和沉积环境}

澄江动物群产于滇东寒武纪筇竹寺期, 约距今 $520 \mathrm{Ma}$ (图 1(b)). 这一地质时期的地层可划分为两个 生物带, 底部的Parabadiella带和上部的EoredlichiaWutingaspis带. 前一个带与玉案山组底部地层相当, 后一个带分布于玉案山组中上部 ${ }^{[1,8,9]}$. 澄江动物群产 出的主要层位为玉案山组帽天山页岩段, 主要分布 在云南东部地区 ${ }^{[911]}$ (图 2). 20 多年来, 大规模的化 石采集、地质调查和化石埋藏学的研究揭示了澄江海口-安宁一带是澄江动物化石保存的核心埋藏相 区 $^{[9]}$, 这一相区帽天山页岩段主要的沉积学特征为灰 绿色薄层泥岩(事件层泥岩)和灰黑色含有机成分高 的泥岩层(背景层泥岩)偶合式叠加组成, 夹有黄色中
层至薄层不等的粉砂岩层, 其中灰绿色薄层泥岩是 软躯体化石保存的主要岩相 ${ }^{[12,13]}$.

众多学者对玉案山组的沉积环境开展了研究, 分别解释为浅海环境 ${ }^{[14]}$, 接近海湾的滨外相 ${ }^{[15]}$ 或三 角洲前缘 ${ }^{[16]}$, 或前滨-近滨环境 ${ }^{[17]}$. 孙枢等 ${ }^{[18,19]}$ 在玉 案山组最早识别出风暴沉积, 对玉案山组的沉积环 境解释提供了依据. $\mathrm{Hu}^{[9]}$ 和 $\mathrm{Zhu}$ 等 ${ }^{[11]}$ 针对富含软躯体 化石保存的帽天山页岩段的沉积结构进行了详细的 描述, 通过埋藏学、沉积学、地球化学等方面的证据 进一步证实了该段岩层沉积于远基滨外至深水滨前 环境, 经常受风暴引起的淡水注入的影响 ${ }^{[11]} . \mathrm{Hu}^{[9]}$ 认 为帽天山页岩段沉积在浅海, 通常泥质基底的陆架 斜坡环境 ${ }^{[1]}$.

\section{2 材料和方法}

本文采用的物种数据是根据 1985 年以来所发表 的澄江动物群化石种类的统计, 包括了 228 个化石物 种数据(图 3, 网络版附表 1(www.earth.scichina.com)). 化石个体丰度数据来源于澄江-海口-安宁地区野外 所获得的化石系统统计，统计中包括了所有能够鉴定 


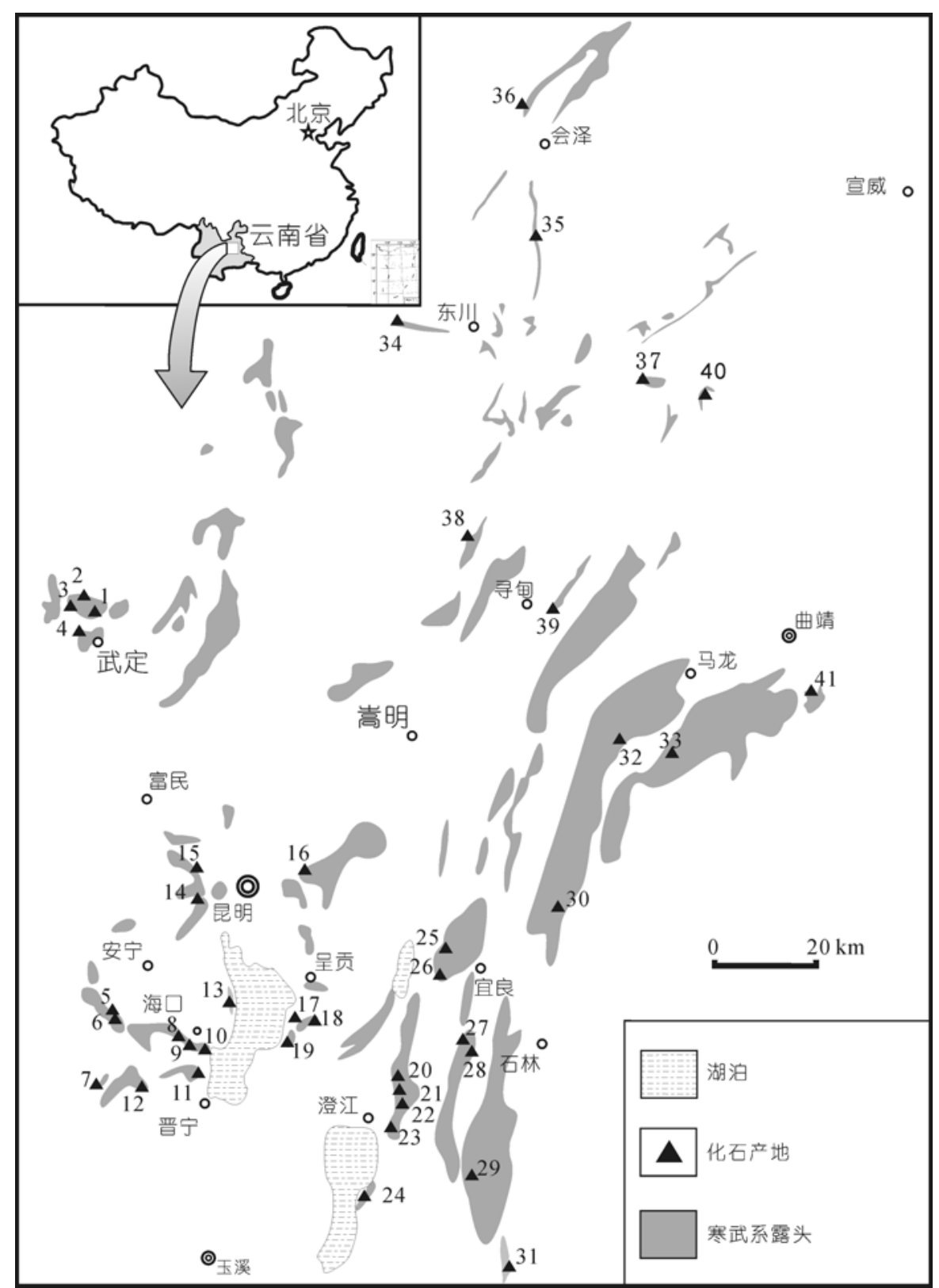

图 2 寒武纪地层在滇东地区分布和澄江动物群化石产地分布图 据 $\mathrm{Hu}^{[9]}$ 修改, 图中数字代表化石产地

的化石个体, 统计数据见网络版附表 2 , 包括 114 个物 种, 18406 个化石个体. 所有的化石样品都保存在中 国科学院南京地质古生物研究所.

我们依据系统古生物学的研究, 将所有物种(除 了疑难和不确定的种类)都归类到门一级的生物分类 单元(图 4, 网络版附表 1). 借鉴Conway ${ }^{[20]}$ 和Caron等 ${ }^{[21]}$ 对布尔吉斯页岩动物群生态功能群的划分方法,
按生活方式和取食方式把澄江动物群物种划分归入 到若干生态功能群(图 5, 网络版附表 1). 依据生物的 生活方式和在水体空间的生活位置, 将把澄江动物 群物种区分为四类: 内栖(infauna sessile, 内栖固着 型, 简写IS; infauna vagile, 内栖活动型, 简写IV)、表 栖(epibenthos sessile, 表栖固着型, 简写ES; epibenthos vagile, 表栖活动型, 简写EV)、中层水游泳 


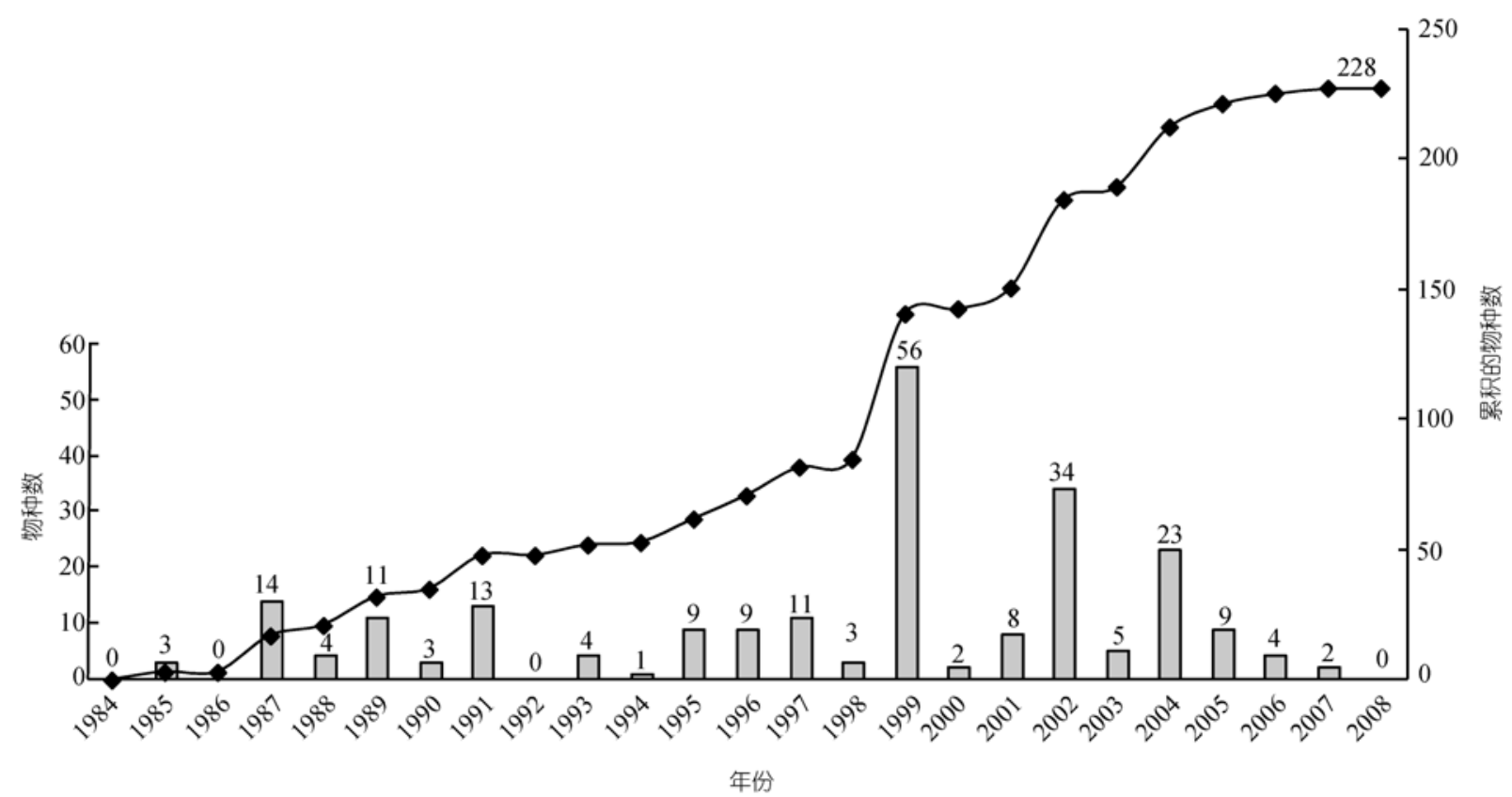

图 3 澄江动物群物种数量累积图

根据每年报道的物种数量

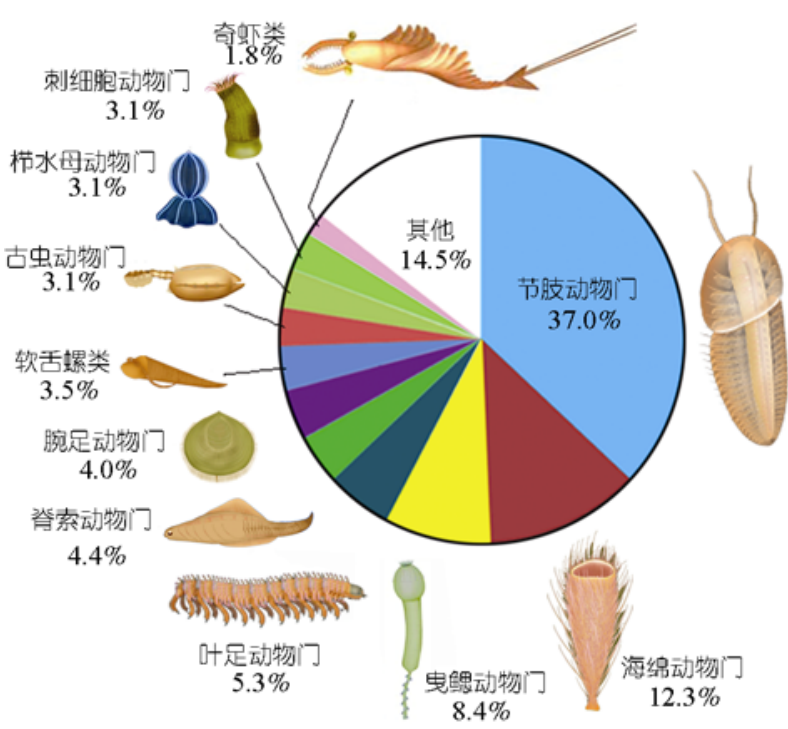

图 4 澄江动物群门类物种丰度饼状图

基于附表 $1, n=228$ 物种

(nektobenthos, 简写 NK) 和浮游 (pelagic, 简写 PE). 依据取食方式, 将把澄江动物群物种区分为以下几 种类型：滤食生物(suspension, 简写 $\mathrm{SU}$ )、食泥生物 (deposit, 简写 DE)、捕食/食腐生物(hunter/scavenger, 简写 HS)、食素生物(grazer, 简写 GR)和杂食生物(om- nivorous, 简写 OM). 藻类等作为初级生产者(epibenthic primary producer, 简写 EPP)对待. 而那些疑难化 石种类, 对其生活方式或取食方式不知晓的, 简写为 UN(unknown). 生活方式和取食方式组合构成了不同 的生态功能群，如 Naraoia spinosa 表栖生活，可自由 活动, 取食方式为食腐, 其所在的生态功能群简写为 EVHS. 每种生物的生活方式和取食方式的确定主要 依据已发表的研究资料. 生态功能群的分类构成了 分析水体生态空间(基底、水/沉积界面、中层水体、 上层水体等空间)物种分异度、群落营养结构、食物 链以及与其他特异化石群对比研究的依据(图 5, 网 络版附表 1).

\section{3 澄江动物群总体物种分异度概述}

迄今为止，澄江动物群中已经有 228 种物种被描 述报道, 它们可以归属于 18 个门一级的生物分类单 元(图 4, 表 1)和 23 个生态功能群(图 5, 表 2). 在寒武 纪早期，丰富多样的门类构成和具有复杂生态功能的 物种大量涌现, 预示着“动物演化树的诞生”, 和生态 空间在寒武纪早期扩张基本完成, 并以此为基础生物 不断适应小生境而使物种间关系变的丰富复杂. 
表 1 澄江动物群门类组成统计表 ${ }^{a)}$

\begin{tabular}{cccc||cccc}
\hline 门类 & 属数 & 种数 & 物种比率 & 门类 & 属数 & 种数 & 物种比率 \\
\hline 藻类 & 3 & 3 & $1.3 \%$ & 刺细胞动物门 & 7 & 7 & $3.1 \%$ \\
节肢动物门 & 75 & 84 & $37.0 \%$ & 奇虾类 & 4 & 4 & $1.8 \%$ \\
海绵动物门 & 21 & 28 & $12.3 \%$ & 棘皮动物门 & 2 & 2 & $0.9 \%$ \\
芈鰓动物门 & 18 & 19 & $8.4 \%$ & 星虫类 & 2 & 2 & $0.9 \%$ \\
叶足类 & 12 & 12 & $5.3 \%$ & 毛顸动物门 & 1 & 1 & $0.4 \%$ \\
脊索动物门 & 10 & 10 & $4.4 \%$ & 环节动物门 & 1 & 1 & $0.4 \%$ \\
腕足动物门 & 9 & 9 & $4.0 \%$ & 开腔骨类 & 1 & 1 & $0.4 \%$ \\
软舌螺类 & 4 & 8 & $3.5 \%$ & 篣虫类 & 1 & 1 & $0.4 \%$ \\
栉水母类 & 7 & 7 & $3.1 \%$ & 分类表明 & 22 & 22 & $9.7 \%$ \\
古虫动物门 & 7 & 7 & $3.1 \%$ & & & & \\
\hline
\end{tabular}

a) 根据发表的物种数据, 见网络版附表 1

表 2 澄江动物群生态类型统计表 ${ }^{a)}$

\begin{tabular}{|c|c|c|c|c|c|}
\hline 生态空间 & 物种数 & 生活方式 & 物种数 & 生态功能群 & 物种数 \\
\hline 浮游 & 12 & & & PEHS & 12 \\
\hline \multirow{5}{*}{ 中层水游泳动物 } & \multirow{5}{*}{26} & & & NKSU & 15 \\
\hline & & & & NKHS & 5 \\
\hline & & & & NK(HS/DE) & 1 \\
\hline & & & & NKDE & 1 \\
\hline & & & & NKUN & 4 \\
\hline \multirow{10}{*}{ 表栖动物 } & \multirow{10}{*}{144} & \multirow{6}{*}{ 表栖活动型 } & \multirow{6}{*}{85} & EVHS & 37 \\
\hline & & & & EVSU & 18 \\
\hline & & & & EV(DE/HS) & 5 \\
\hline & & & & EVOM & 4 \\
\hline & & & & $\mathrm{EV}(\mathrm{DE} / \mathrm{SU})$ & 1 \\
\hline & & & & EVUN & 20 \\
\hline & & \multirow{4}{*}{ 表栖固着型 } & \multirow{4}{*}{59} & ESSU & 43 \\
\hline & & & & ES(GR/DE) & 8 \\
\hline & & & & ES(HS/SU) & 2 \\
\hline & & & & ESUN & 6 \\
\hline \multirow{7}{*}{ 内栖动物 } & \multirow{5}{*}{27} & \multirow{4}{*}{ 内栖活动型 } & \multirow{4}{*}{26} & IVHS & 15 \\
\hline & & & & IVDE & 6 \\
\hline & & & & IVSU & 4 \\
\hline & & & & IVUN & 1 \\
\hline & & 内栖固着型 & 1 & ISHS & 1 \\
\hline & \multirow{2}{*}{\multicolumn{2}{|c|}{$\begin{array}{c}\text { 分类不明 } \\
\text { 初级生产者 }\end{array}$}} & & UNUN & 16 \\
\hline & & & & EAPP & 3 \\
\hline
\end{tabular}

a) 基于发表的物种数据, 见网络版附表 1

\section{1 生物门类组成}

澄江动物群的物种分异度高(228 种), 多数物种 为单属种, 通常具有门一级分类特征. 已经确定的生 物门类 18 个(图 4, 表 1), 按各类物种所占比例, 不同 门类在动物群中的顺序依次是节肢动物 (arthropods, 84 种, 占 $37 \%$ )、海绵动物(poriferans, 28, 12.3\%)、曳 鳃动物(priapulids, 19, 8.4\%)、叶足类(lobopods, 12, $5.3 \%$ )、脊索动物 (chordates, $10,4.4 \%$ )、腕足动物 (brachiopods, 9, 4\%)、软舌螺(hyoliths, $8,3.5 \%$ )、古虫 动物 ${ }^{[22]}$ (vetulicolids, $7,3.1 \%$ )、栉水母动物(ctenoph- ores, 7, 3.1\%)、刺细胞动物(cnidarians, 7, 3.1\%)、奇虾 类(anomalocarids, 4, 1.8\%)、藻类(algae, 3, 1.3\%)、棘 皮动物? ${ }^{[23]}$ (echinoderms, 2, $0.9 \%$ )、星虫动物 (sipunculas, 2, 0.9\%)、毛颚类动物(chaetognaths, 1, $0.4 \%$ )、环节动物(annelids, $1,0.4 \%$ )、开腔骨类(chancellorids, $1,0.4 \%$ )、篣虫动物(phoronids, 1, 0.4\%), 还 有大量疑难化石其生物门类属性难以判定(unknown, $22,9.7 \%)$. 上述数据表明, 澄江动物群不仅包括了现 生海洋中主要的无脊椎动物门类, 而且还出现了原 始的脊椎动物(vertebrates), 如昆明鱼(Myllokunm- 


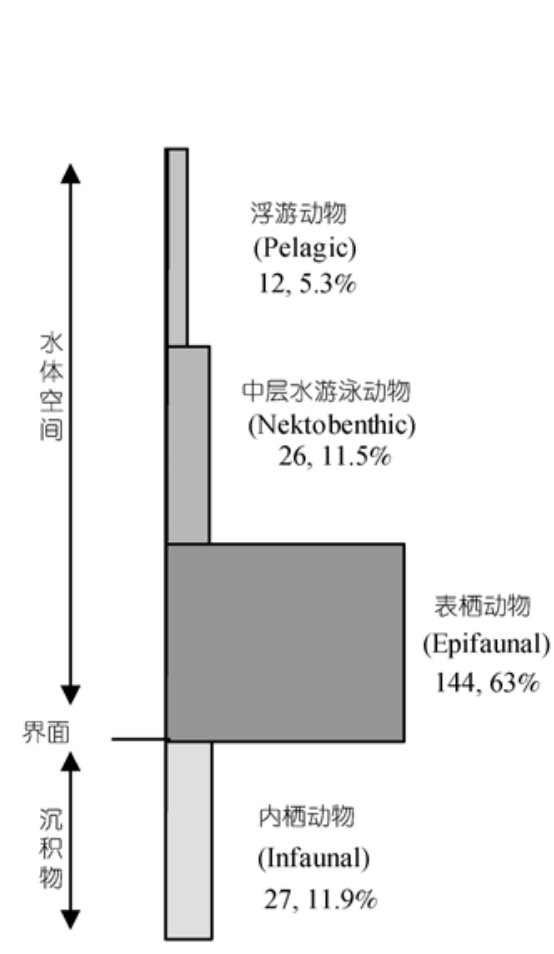

(a)

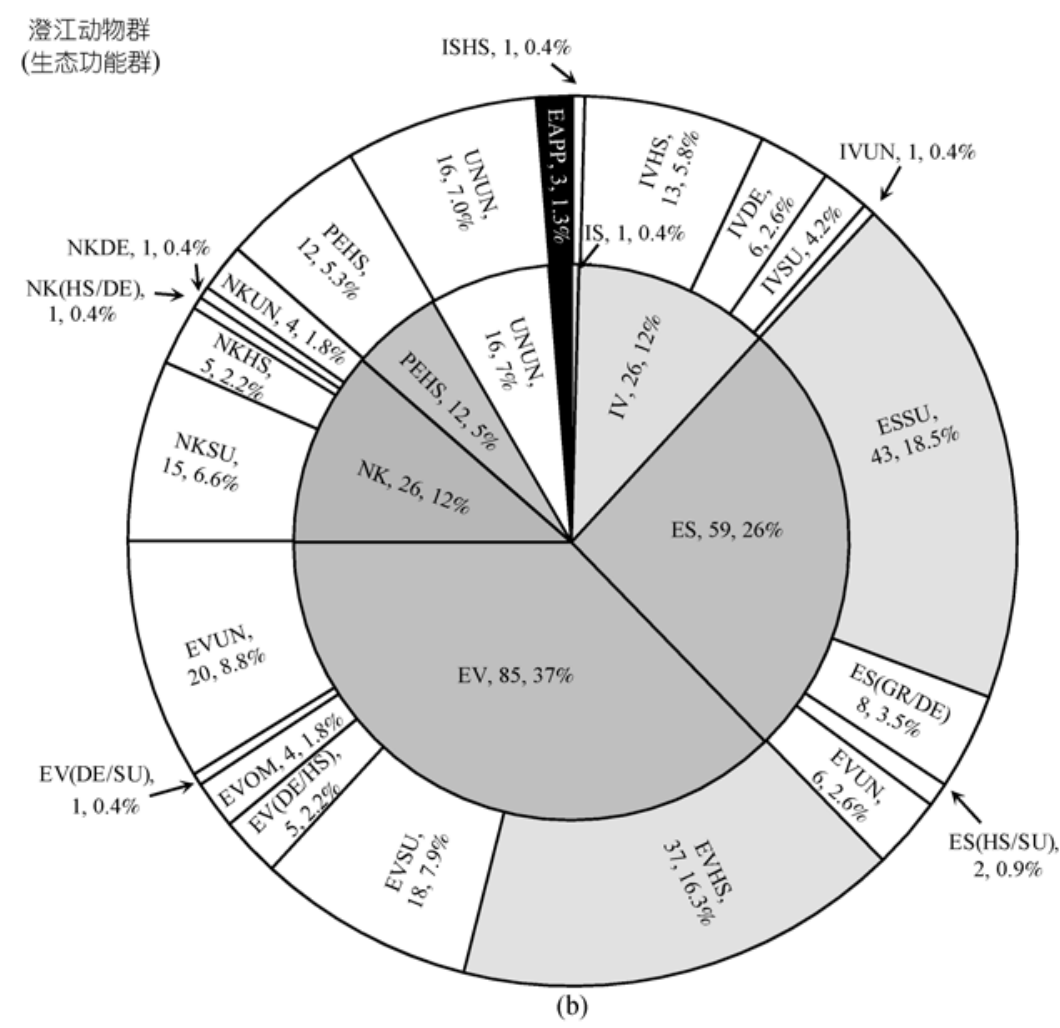

图 5 澄江动物群生态功能群丰度图

基于网络版附表 1. (a) 水体生态空间物种分异度; (b) 生态功能群相对物种丰度. 字母组合含义见文中解释

ingia fengjiaoa)、海口鱼(Haikouichthys ercaicunen-

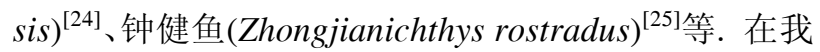
们的统计分析中, 节肢动物相对丰度最高, 占物种数 量的 $37 \%$, 并不像先前学者Leslie等 ${ }^{[26]}$ 和 $\mathrm{Hou}$ 等 ${ }^{[3]}$ 所 报道的 $60 \%$. 究其原因主要是因为近年来大量新化 石点的发现和持续的化石发掘, 新的软躯体化石种 类不断被发现，使节肢动物所占比值下降. 还值得一 提的是, 在澄江动物群里除现生的生物门类外, 还包 括一些早以灭绝的动物门类, 如古虫动物门 (vetulicolids), 很可能是后口动物谱系基干类群 ${ }^{[22,27]}$. 同时, 后口动物谱系中的原始脊椎动物 (e.g. Myllokunmingia fengjiaoa, Haikouichthys ercaicunensis $)^{[24]}$ 以及原始棘皮动物古囊类(echinoderms? Dianchicystis jianshanensis, Vetulocystis catenata $)^{[23]}$ 、具头索动物特

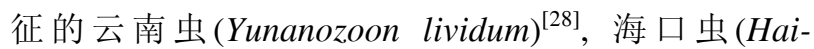
kouella lanceolata) ${ }^{[29]}$ 以及尾索动物 (Shankouclava anningense ${ }^{[30]}$, Cheungkongella ancestralis $\left.{ }^{[31]}\right)$ 的化石 代表都在澄江化石库里发现, 因而早期动物演化谱
系图在寒武纪早期基本构建完成 ${ }^{[6]}$. 近年来新的化石 证据也证实了埃迪卡拉生物群分子在澄江化石库也 有保存 (Stromatoveris psygmoglena $)^{[32]}$. 以上分析数 据, 充分支持了动物在寒武纪爆发式辐射演化事件 的规模和特征.

\section{2 生态功能群组成}

图 5 和表 2 显示了澄江动物群物种生态功能群组 成关系. 由此可以看出, 表栖动物主控了整个生物群 落(epibenthos, 144 种, 63\%), 接下来依次是底内动物 (infaunal organisms, 27, 11.9\%)和中层水游泳的物种 (nektobenthos, 26, 11.5\%), 具浮游物种数量较少 (pelagic organisms, 12, 5.3\%). 中层水游泳和浮游生 物在澄江动物群所占的比例少, 可能与埋藏偏差有 直接关系, 因为它们生活的位置远离洋底处于水层 上部或顶部，风暴引起的远基泥质絮状沉积物沉降 范围并不能有效的达到其主要的生活水层区域，而 底栖物种或处于水层下部的物种在絮状沉积物沉降 
范围内, 可以有效的被捕获, 这可能是澄江动物群以 底栖物种为主的原因之一. 从图 5 中可以看出, 动物 群中以表栖活动型 $(\mathrm{EV}, 85,37.4 \%)$ 和表栖固着型( $\mathrm{ES}$, $59,25.6 \%)$ 生物为主. 生活在沉积物环境里的内栖物 种多数为活动型 (IV, 26, 12\%), 固着生活方式较少(IS, $1,0.4 \%)$. 动物的取食方式多数为滤食型( $\mathrm{SU}, 35.6 \%)$ 和捕食/食腐(HS, $31.1 \%$ ). 生态功能群以表栖固着滤 食类(ESSU，43，18.5\%)和表栖活动捕食或食腐类为 主(EVHS, 37, 16.3\%), 其他生态功能群按比例依次为 表栖活动滤食类(EVSU, 18, 7.9\%)、底栖游泳滤食类 (NKSU, 15, 6.6\%)、内栖活动捕食类(IVHS, 13, 5.8\%) 和浮游捕食类(PEHS, 12, 5.3\%). 其他生态功能群 (ISHS, 0.4\%; IVDE, 2.6\%; IVSU, 2\%; ESGR/DE, 3.5\%; ESHS/SU, $0.9 \%$; EVDE/HS, 2.2\%; EVOM, $1.8 \%$; EVDE/SU, 0.4\%; NKHS, 2.2\%; NKHS/DE, 0.4\%; NKDE, $0.4 \%$ )仅占整个澄江动物群的 $16.8 \%$. 具有不 确定生活方式或取食方式的生态功能群(包括IVUN, 0.4\%; ESUN, 2.6\%; EVUN, 8.8\%; NKUN, $1.8 \%$; UNUN, $7 \%$ ) 占整个群落 $20.6 \%$. 以上定量分析表明, 澄江动物群物种具有丰富多样的生活方式, 已经占 据生态空间不同空间, 如从沉积物内部到水体不同 高度. $\mathrm{Hu}$ 等 ${ }^{[5]}$ 曾对澄江动物群的浮游物种进行过归纳 总结, 认为澄江动物群中不同的浮游物种具有复杂 多样的取食策略, 指示了在寒武纪早期浮游群落已 经演化出具现代海洋的食物网结构. 值得注意的是, 我们的分析表明整个生态系统营养结构在寒武纪早 期就可能非常复杂, 因为大多数物种都具有滤食 $(\mathrm{SU}$, 35.6\%)和捕食或食腐取食策略(HS, 31.1\%). 尽管这 种以捕食与被捕食关系形成的层次相对简单, 但足 可以预示着早期食物网已经演化出不同的营养级结 构, 指示具有多层次营养级结构的金字塔式的食物 网在寒武纪早期已经形成.

\section{4 澄江动物群物种个体丰度模式分析}

\section{1 总体丰度特征}

我们获得的个体丰度数据包含 114 个物种, 它们 归属 12 个动物门或相对于门一级的分类单元和 17 个 生态功能群. 所有物种个体丰度数据按其大小进行 排序展示在图 6 中, 个体丰度比例小于 $1 \%$ 的物种没 有在图中显示. 从图 6 可以看出, 澄江动物群共有 21 个物种占到总物种数的 $18.4 \%$, 但它们的个体数量占
整个个体数量的 $89.3 \%$. 数量最多的物种是节肢动物 的Kunmingella douvillei (占整个个体数量 $26.2 \%$ )、曳 鳃动物的Cricocosmia jinningensis (15.4\%)、腕足动物 的 Diandongia pista (11\%), 其次是节肢动物的 Leanchoilia illecebrosa (4.9\%) 和曳鳃动物的 Mafangscolex sinensis (4.2\%), 其余的 109 个物种的个 数数量总和仅占 $38.3 \%$. 由此可见, 物种个体数量集 中分布在少数物种上. 采用物种个体丰度以对数 2 排 序, 检测每个数量级内的物种数量, 结果显示物种数 量的丰度级序列分布很符合对数正态分布. 使用 Kolmogorov-Smirnow 拟合计算 ${ }^{[33]}$, 显示了期望值分 布与观察数据非常高的吻合程度(图 7). 这个与Caron 等 ${ }^{[21]}$ 对布尔吉斯页岩的个体丰度与物种数也呈对数 正态分布的结果一致, 指示了这两个寒武纪保存最 完整的动物群落具有相同的丰度模式.

\section{2 不同生物门类的物种丰度模式}

野外采集的化石数据(114 物种, 18406 个体)和文 献统计物种数据(228 物种)对比, 两组数据所显示的 各门类物种丰度大小排序相似, 节肢动物门、海绵动 物门、曳鳃动物门、叶足动物门、腕足动物门是物种 丰度最高的前五个门类(表 3). 个体丰度最高的门类 是节肢动物门、曳鰓动物门、腕足动物门, 分别占总 个体数的 $51.8 \%, 22.6 \%$ 和 $16.3 \%$ (图 8), 其余门类个 体数量总和仅占到 $9.3 \%$. 数据揭示了无论从个体丰 度还是物种丰度上来看节肢动物都主控整个群落. 大部分门类的个体丰度和物种丰度排序不一致, 这 个结论可以从个体和物种相对丰度两个方面排序更 清楚的看出, 如海绵动物门物种分异度很高, 排序位 于第二位, 但个体丰度却不高, 仅排到第六位, 相对 个体丰度也仅有 $1.2 \%$ (表 3). 叶足动物也具有相同特 点, 物种分异度相对很高, 位于第三位, 而个体丰度 仅排到第七位, 曳鳃动物门具有相近的排序级, 物种 丰度位于第三位, 个体丰度位于第二位. 腕足动物门 个体丰度排序要比物种丰度排序高(表 3).

个体丰度最高的三个门类(节肢动物、曳鳃动物、 腕足动物)的代表物种具体分析显示(见图 9), 节肢动 物中 Kunmingella douvillei 个体最多, 占到整个节肢 动物的 $51.3 \%$, 其次是 Leanchoilia illecebrosa $(9.5 \%)$, Naraoia longicaudata(6\%)和 Naraoia spinosa (5.1\%) 


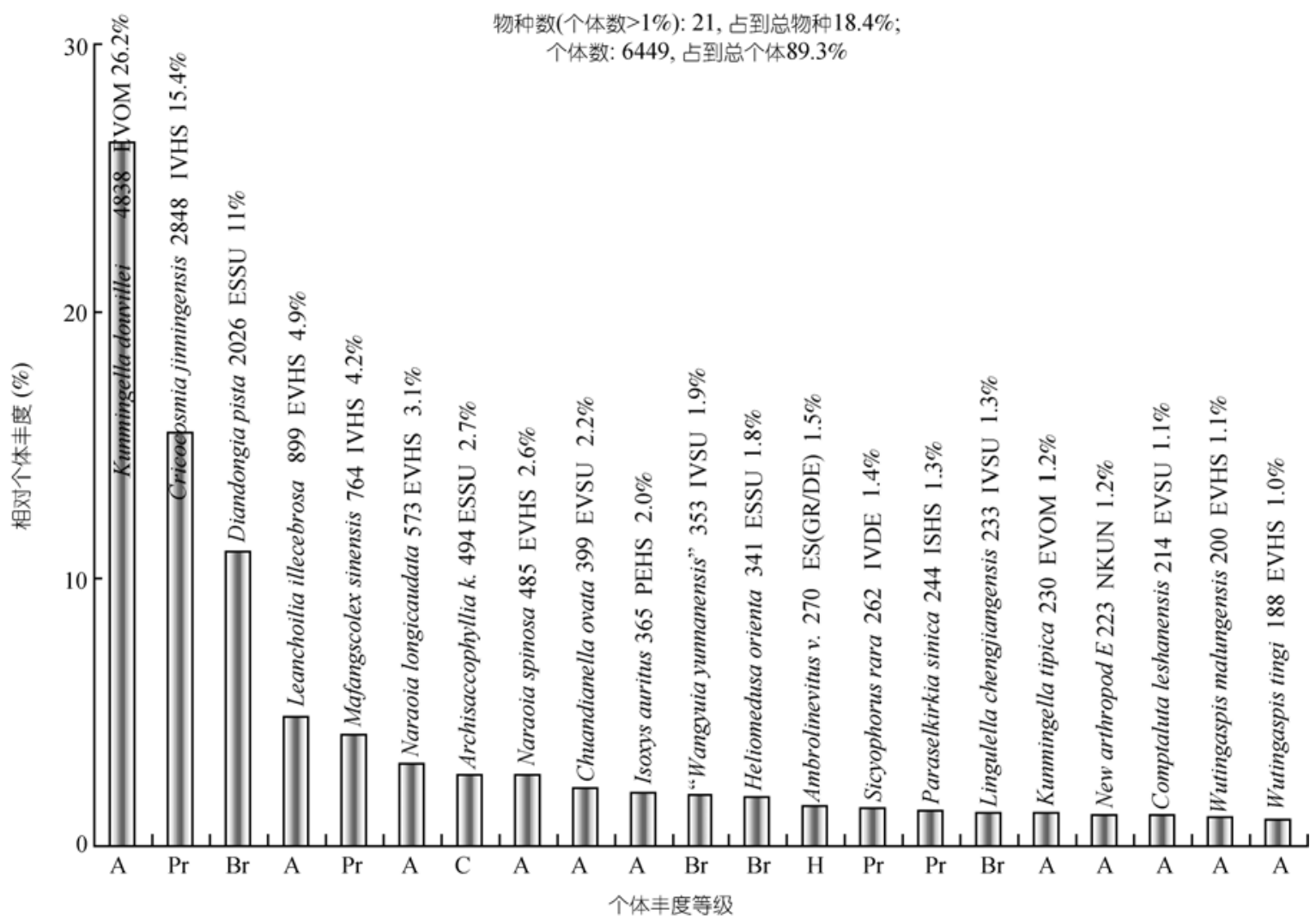

图 6 对比澄江动物群物种个体丰度

基于采集的化石个体丰度数据(见网络版附表 2), 图中标示出物种名称、所归属的生物门类、生态群、个体数量及所占的百分比, 图中只列 出个体丰度大于 $1 \%$ 的物种共 21 种占采样物种总数的 $18.4 \%$, 但其个体数量占到整个采集样品的 $89.3 \%$. A, 节肢动物; $\mathrm{Pr}$, 曳鳃动物; $\mathrm{Br}$, 腕 足动物 $; \mathrm{C}$, 刺细胞动物; $\mathrm{H}$, 软舌螺类

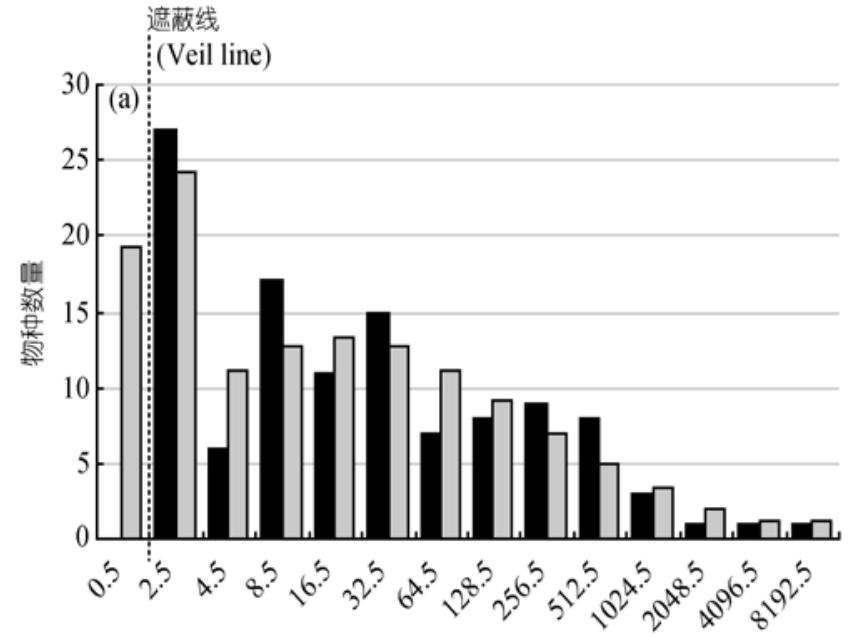

个体数量等级

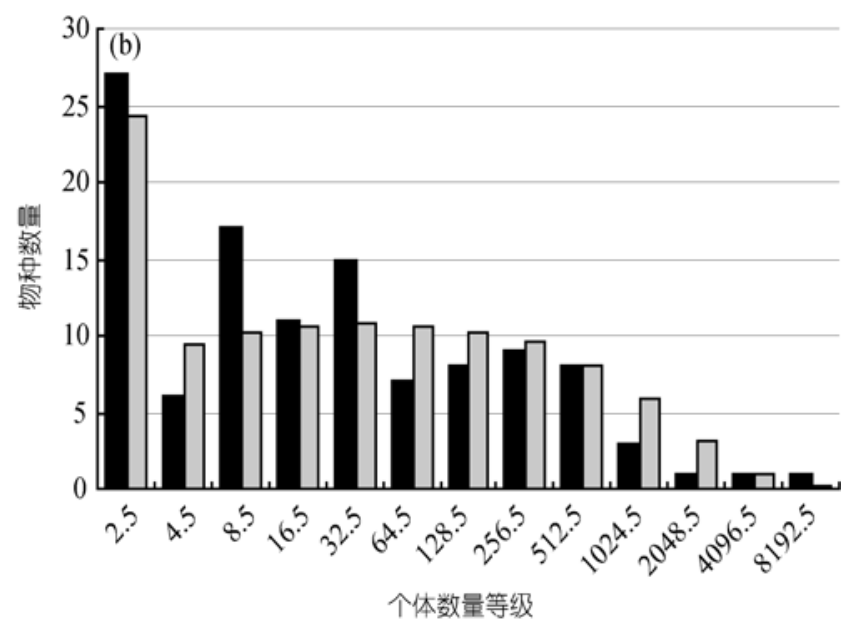

个体数量等级

图 7 Kolmogorov-Smirnov 拟合优度检验图

黑色为样品中实际观察值; 灰色为理论计算值, 方法解释见Magurran ${ }^{[33]}$. (a) 截尾对数正态分布; (b) 对数级数分布 

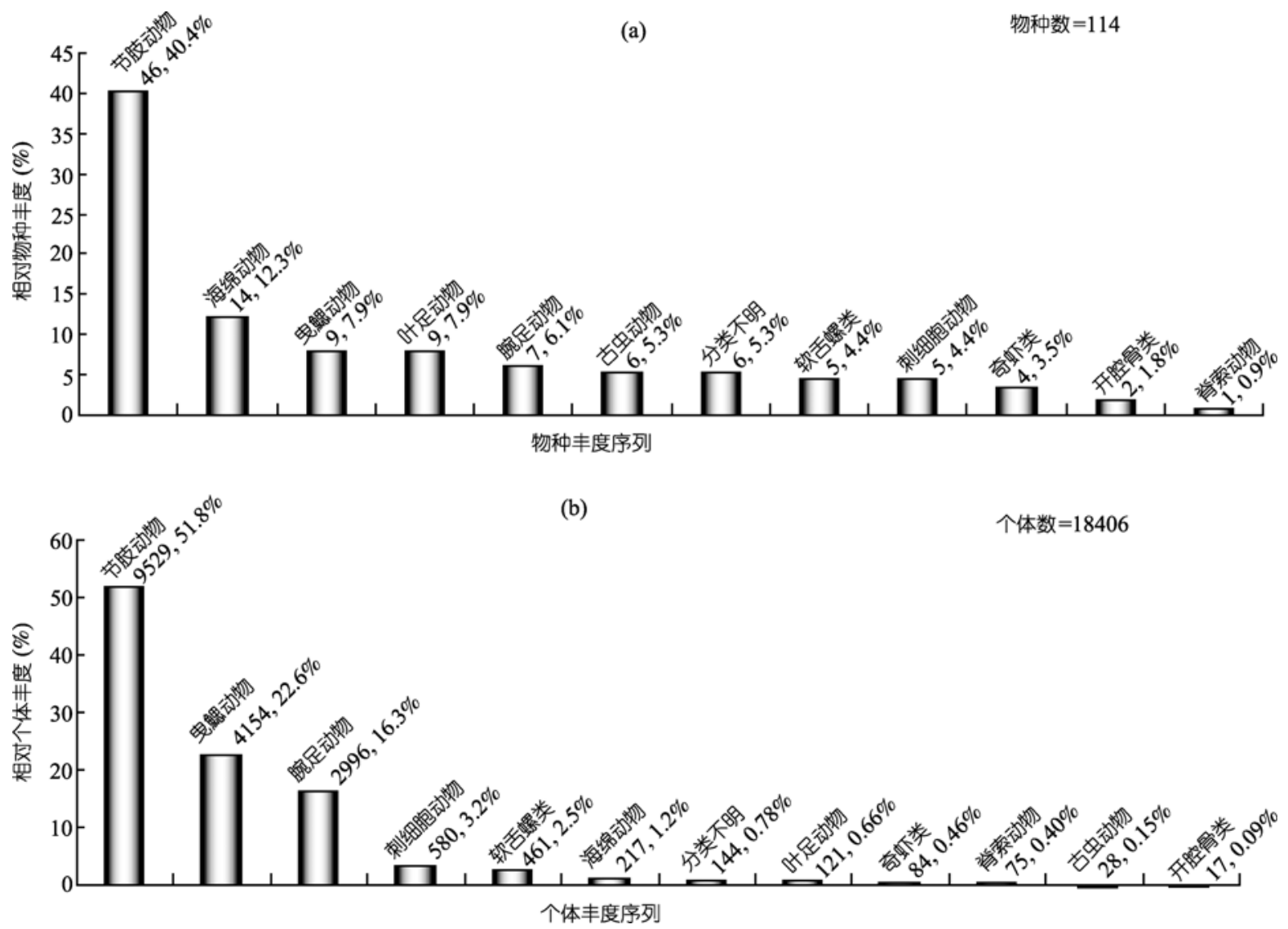

图 8 澄江动物群门类相对物种丰度和个体丰度柱状图

(a) 物种; (b) 个体, 数据来源于网络版附表 2

表 3 澄江动物群物种多样性统计表

\begin{tabular}{|c|c|c|c|c|c|c|c|c|}
\hline \multirow[b]{2}{*}{ 动物门类 } & \multicolumn{3}{|c|}{ 基于文献统计的数据 } & \multicolumn{5}{|c|}{ 野外采集统计的个体丰度数据 } \\
\hline & 物种数 & $\begin{array}{c}\text { 物种比率 } \\
(\%)\end{array}$ & $\begin{array}{c}\text { 排序 } \\
\text { 物种丰度 } \\
\end{array}$ & 物种数 & $\begin{array}{c}\text { 物种比率 } \\
(\%)\end{array}$ & $\begin{array}{c}\text { 排序 } \\
\text { 物种丰度 }\end{array}$ & $\begin{array}{c}\text { 个体丰度 } \\
(\%)\end{array}$ & $\begin{array}{c}\text { 排序 } \\
\text { 个体丰度 } \\
\end{array}$ \\
\hline 节肢动物门 & 84 & 37 & 1 & 46 & 40.3 & 1 & 51.8 & 1 \\
\hline 海绵动物门 & 28 & 12.3 & 2 & 14 & 12.3 & 2 & 1.2 & 6 \\
\hline 曳鰓动物门 & 19 & 8.4 & 3 & 9 & 7.9 & 3 & 22.6 & 2 \\
\hline 叶足类 & 12 & 5.3 & 4 & 9 & 7.9 & 3 & 0.66 & 7 \\
\hline 腕足动物门 & 9 & 4 & 6 & 7 & 6.1 & 5 & 16.3 & 3 \\
\hline 古虫动物门 & 7 & 3.1 & 8 & 6 & 5.3 & 6 & 0.15 & 10 \\
\hline 软舌螺类 & 8 & 3.5 & 7 & 5 & 4.4 & 7 & 2.5 & 5 \\
\hline 刺细胞动物门 & 7 & 3.1 & 8 & 5 & 4.4 & 7 & 3.2 & 4 \\
\hline 奇虾类 & 4 & 1.8 & 11 & 4 & 3.5 & 9 & 0.46 & 8 \\
\hline 开腔骨类 & 1 & 0.4 & 14 & 2 & 1.8 & 10 & 0.09 & 11 \\
\hline 脊索动物门 & 10 & 4.4 & 5 & 1 & 0.9 & 11 & 0.4 & 9 \\
\hline
\end{tabular}

(图 9(a)). 曳鳃动物中以 Cricocosmia jinningensis 个 体为最多, 占到整个曳鳃动物的 $68.5 \%$, 其次是 Mafangscolex sinensis(18.4\%), Sicyophorus rara(6.3\%) 和 Paraselkirkia sinica (5.9\%)(图 9(b)). 腕足动物中
Diandongia pista 个体最多, 占到整个腕足动物的 $67.6 \%$, 其次是 Wangyuia yunnanensis(11.8\%), Heliomedusa orienta(11.4\%)和 Lingulella chengjiangensis $(7.8 \%)$ (图 9(c)). 结果显示不同门类中物种的个体丰 

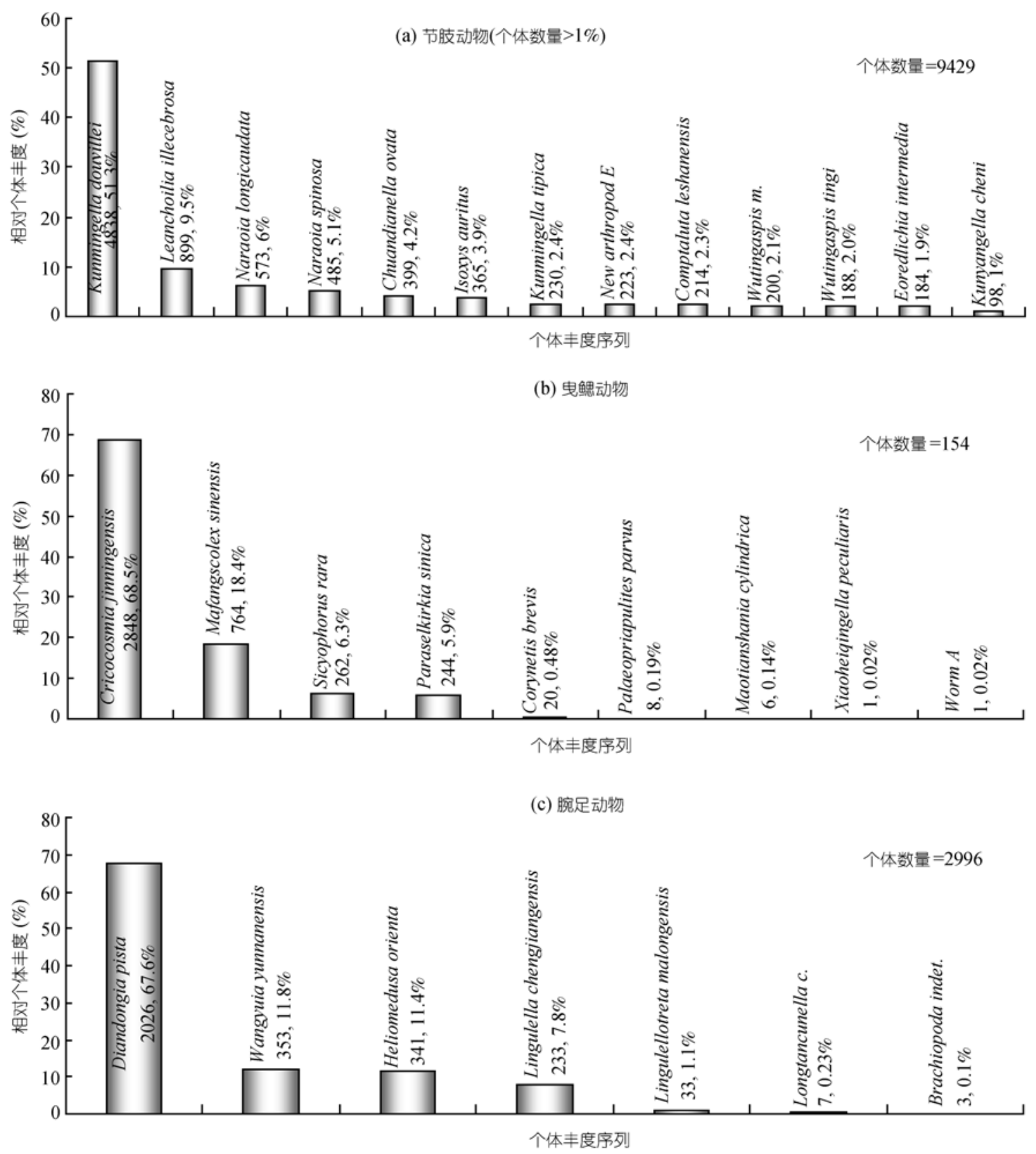

图 9 对比澄江动物群个体数量最多的三个生物门的物种个体丰度和物种代表

度分布具有不均衡特征, 极少数物种主控着各个门 类, 也就是说澄江动物群中各门类具有相对低的均 匀度.

我们选用Whittaker图点方法来进一步分析主要 各门类间物种个体丰度的分布特征. Whittaker ${ }^{[34]}$ 图点 方法也被称为个体丰度等级 (Rank-abundance) 图点或 优势度/多样性(dominance/diversity)曲线, 被设计用
于表述物种丰度和各物种个体数量相对比例的均匀 程度, 即均匀度. 均匀度在这里被定义为群落分类单 元内物种的个体丰度分布模式，即种间个体比例分 配的程度. 如果均匀度低(分类单元内具有相对少的 物种组成, 个体数量主要集中在少数物种上), Whittaker 图点形成的线性回归线显示为一条倾斜度 高的直线, 极端情况为一条垂直线. 相反, 如果均匀 
度高(各物种个体数量相对均衡), 图点所形成的线性 回归线为一条倾斜度低的直线, 极端情况为一条水 平线. Whittaker图点二维图示方法为: 物种按其个体 数量从大到小在 $X$ 坐标轴上排序, 各物种个体数量值 由 $\lg$ 转换后, 其值在 $Y$ 轴上表示, 根据这些点形成线 性回归线, 其斜率用于表述群落的均匀度. 个体数量 的对数转换后不会影响各物种的相对丰度. 这种转 换后最大好处是可以有效地减少某些物种的绝对丰 度对计算结果的影响. 方法的解释进一步见 Magurran ${ }^{[33]}$ 解释.

从澄江动物群中物种丰度前五的动物门(节肢动 物门、海绵动物门、曳鳃动物门、叶足动物门、腕足 动物门)的 Whittaker 图点可以看出, 节肢动物门具有 最高均匀度, 曳鳃动物门和腕足动物门虽然有很高 的个体丰度, 但它们的均匀度比海绵动物门和叶足 动物门都低, 预示个体数量明显集中在少数物种上 (图 10).

\section{3 不同生态功能群的丰度模式}

澄江动物群中不同生态功能群的个体丰度模式 见图 11 和表 4. 表栖活动捕食/食腐类(EVHS)和表栖 固着滤食类 $(\mathrm{ESSU})$ 是物种丰度最高的类型, 这种结 论与根据文献统计物种数据是一致的(图 5(b), 11(a)).
个体丰度最高的生态功能群是表栖活动杂食类 $(\mathrm{EVOM}, 28.2 \%)$ 、底内活动捕食/食腐类(IVHS, $19.8 \%$ )、表栖固着滤食类(ESSU, 17.7\%)和表栖活动捕 食/食腐类(EVHS, $15.3 \%)$. 这四个生态功能群个体数 量占到整个群落 $81 \%$. 其他的生态功能群个体数量 所占比例都很少, 都没有超过 4.4\%(图 11(b)). 从四 类生态群的物种组成分析可以看出, EVOM 主要以节 肢动物 Kunmingella douvillei 为主, 其个体数占到该 类型的 $93.2 \%$ (图 12(a)); IVHS 主要以曳鳃动物 Cricocosmia jinningensis 为主, 其个体数占到该类型 的 78\%, 其次是曳鳃动物 Mafangscolex sinensis 占到 20.9\%(图 12(b)). 这两种生态类型个体数量高, 但其 物种数量所占比例并不高, 仅占到 4.4\%和 7\%(图 11). ESSU 和 EVHS 的物种数量所占的比例明显比前两类 高(图 11(a)), ESSU 中个体丰度最高的物种是腕足动 物 Diandongia pista 占到该类群的 $62 \%$, 其次是刺细 胞动物 Archisaccophyllia kunmingensis(15.1\%)和腕足 动物 Heliomedusa orienta(11\%)(图 12(c)); EVHS 中个 体数量最多的是节肢动物 Leanchoilia illecebrosa, Naraoia longicaudata 和 Naraoia spinosa 分别占到该 类群的 32\%,20\%, 17\%(图 12(d)). 丰度最高的四类生 态功能群的 Whittaker 图点显示 EVOM 和 IVHS 的均 匀度远低于 ESSU 和 EVHS, 但相对于 ESSU 和 EVH,

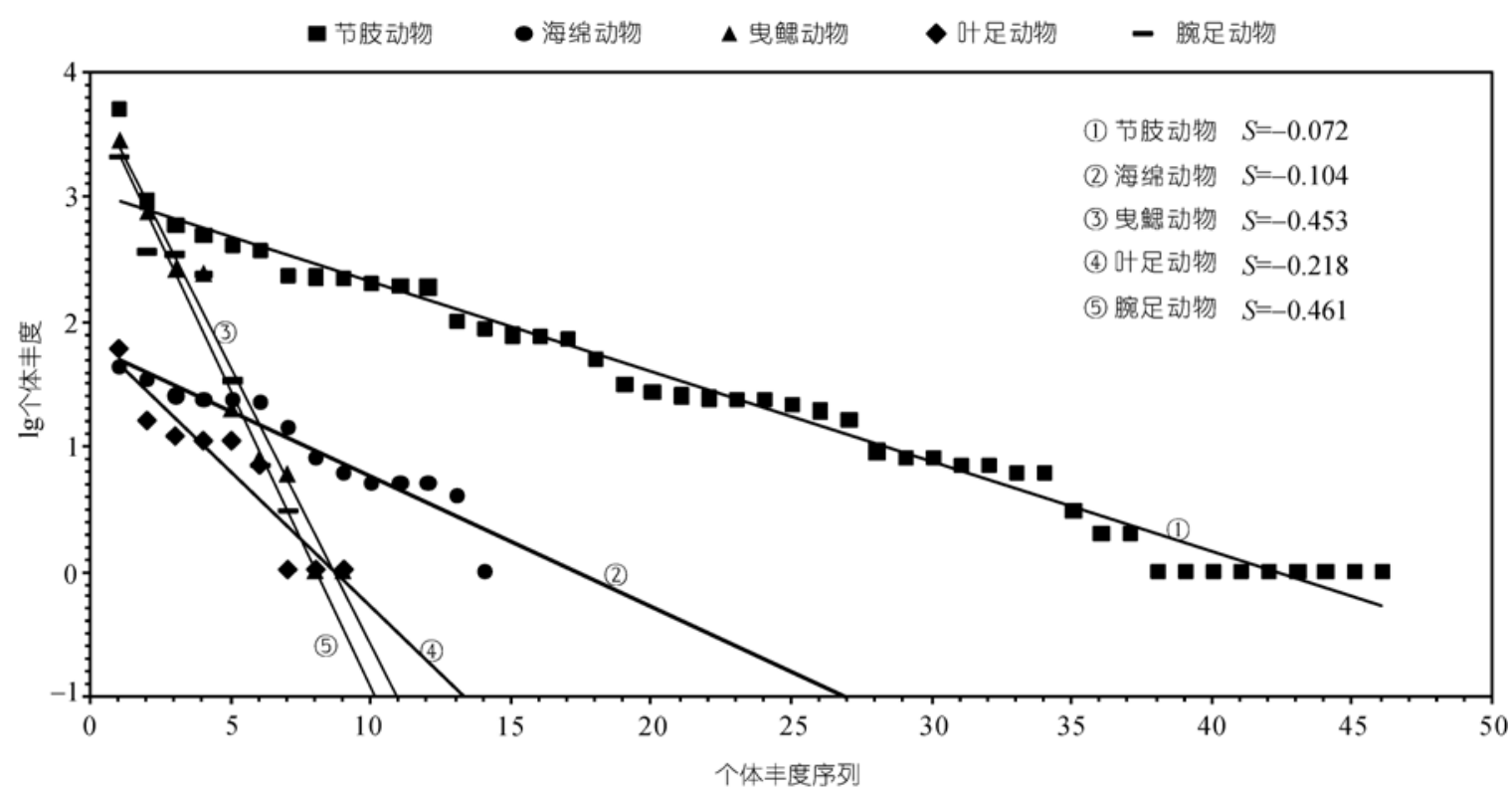

图 10 物种最丰富的五类动物门的 Whittaker 点图

图中标注出每一类点集形成的线性回归线和斜率值 

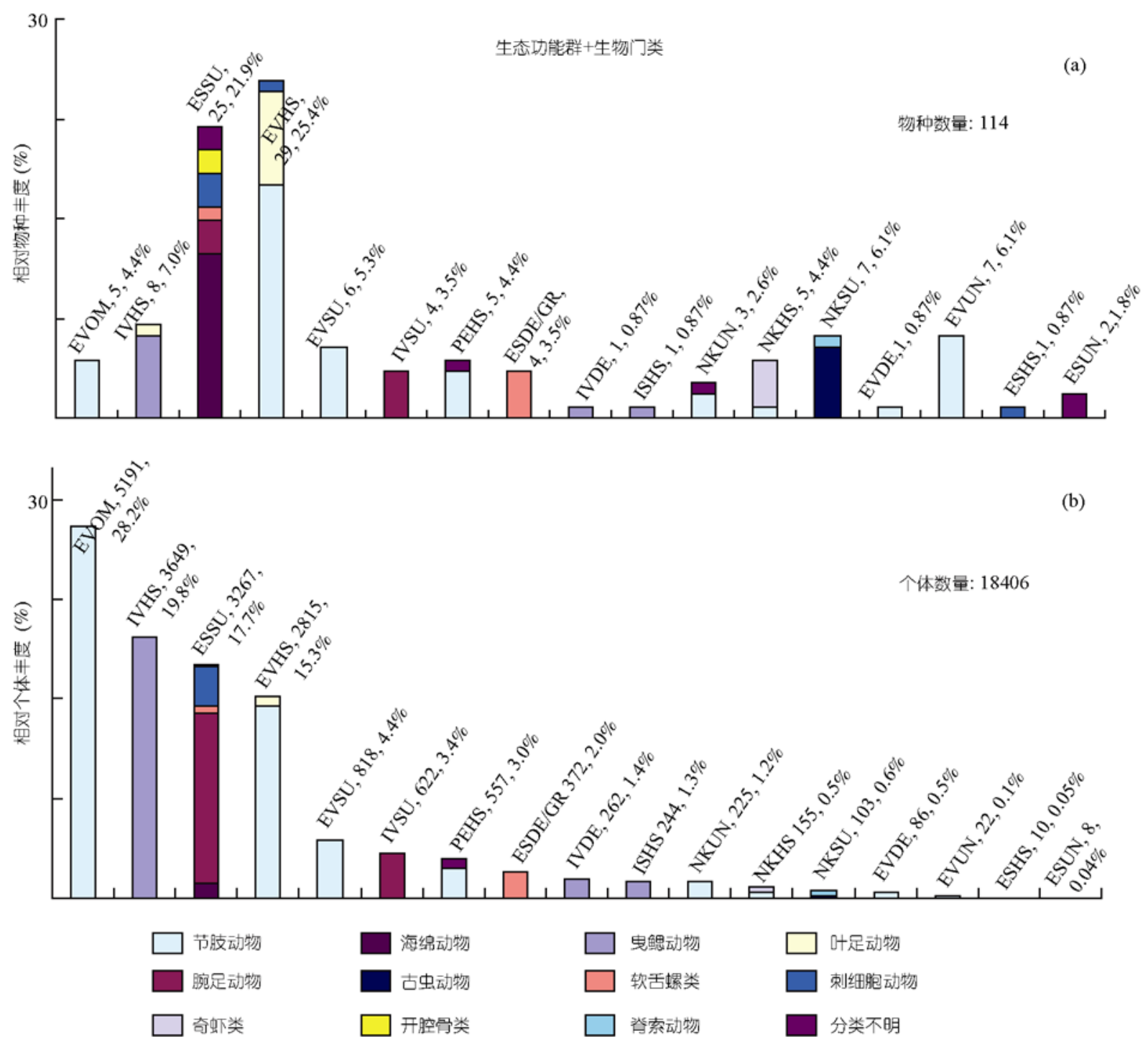

(b)

个体数量: 18406

图 11 对比澄江动物群生态群物种丰度和个体丰度

(a) 物种; (b) 个体. 数据来源于野外采集的数据, 见网络版附表 2

EVOM, IVHS 具有高的个体数量和低的物种多样性 (图 13).

澄江动物群动物取食策略可区分为捕食或食腐 $(\mathrm{HS}) 、$ 滤食 $(\mathrm{SU}) 、$ 食沉积物 $(\mathrm{DE})$ 和杂食 $(\mathrm{OM})$ 等, 其中 营滤食和捕食/食腐生活的动物在澄江动物群中占到 主要地位, 物种数量都超过了 $30 \%$ (表 5). 捕食或食 腐者类型个体数量占到 $40.4 \%$, 其次是杂食者个体数 量有 $28.2 \%$. 但杂食者的物种数量却很少, 只占到物 种数的 $1.4 \%$. 滤食者也有较多的个体数量, 其百分 比为 $26.1 \%$ (表 5).
从不同水体生态空间内的个体数量来看，澄江 动物群中表栖动物均匀度要远高于浮游动物、底内动 物和中层水游泳动物, 中层水游泳动物均匀度稍高 于底内动物和浮游动物, 而后两者均匀度相似(图 14). 结果指示了澄江动物群主要以表栖动物 (ES 和 $\mathrm{EV}$ )为 主, 它们不但物种多, 而且个体数量也最多占到整个 个体数量的 $68.4 \%$ (ES 为 $48.5 \%$, ES 为 $19.9 \%$ ), 其次 是底内动物个体数量占 $25.9 \%$ (IV 为 $24.6 \%$, IS 为 $1.3 \%$ (表 4). 浮游动物、中层水游泳动物与底内动物 具有相似的均匀度, 从物种数量来看浮游动物和中 


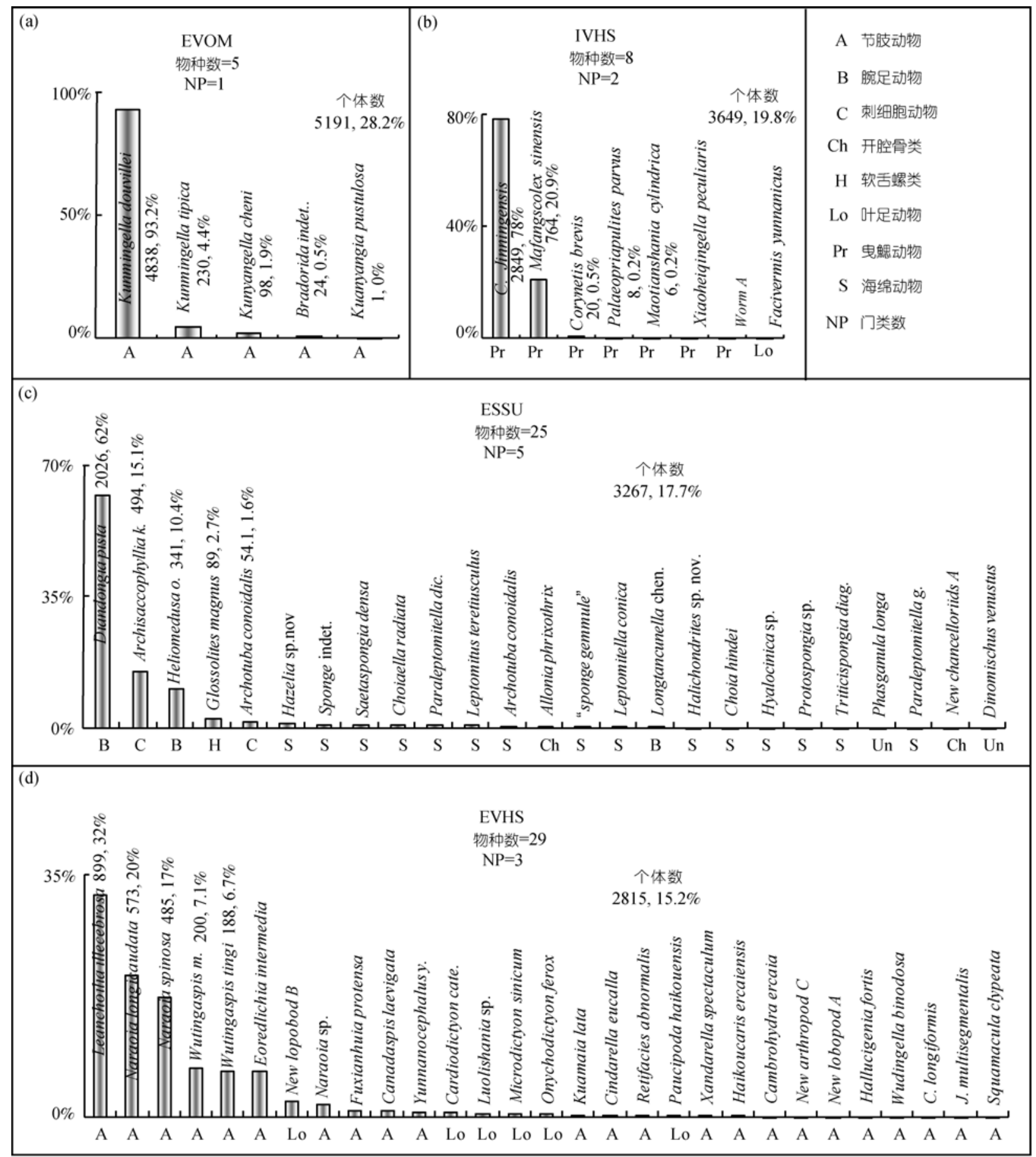

图 12 对比澄江动物群个体数量最多的四个生态群的物种个体丰度

生态功能群分类字母所代表的含义见网络版附表 1

层水游泳动物并不比底内动物少很多, 而且中层水 游泳物种数量与底内动物相当(表 2,4 ), 但它们的个 体数量相差很大. 这种结果有可能与澄江动物群埋
藏方式有关. 因为产化石沉积围岩主要形成于风暴 引起的悬浮絮状物的快速沉积, 如果风暴流不能有 效的达到浮游动物和中层水游泳动物生活的主要水 
表 4 澄江动物群生态类型统计表 ${ }^{a)}$

\begin{tabular}{|c|c|c|c|c|c|c|}
\hline \multicolumn{2}{|c|}{ 生态空间(物种数) } & 生态功能群 & 个体数量 & 物种数量 & 多样性指数 & 个体丰度 $(\%)$ \\
\hline 浮游(5) & $\mathrm{PE}(5)$ & PEHS & 557 & 5 & -0.356 & 3.1 \\
\hline \multirow{3}{*}{ 中层水游泳(15) } & \multirow{3}{*}{$\mathrm{NK}(15)$} & NKSU & 103 & 7 & \multirow{3}{*}{-0.169} & \multirow{3}{*}{2.6} \\
\hline & & NKHS & 155 & 5 & & \\
\hline & & NKUN & 225 & 3 & & \\
\hline \multirow{9}{*}{ 表栖(80) } & \multirow{4}{*}{$\mathrm{ES}(32)$} & ESSU & 3267 & 25 & \multirow{9}{*}{-0.037} & \multirow{9}{*}{68.4} \\
\hline & & $\mathrm{ES}(\mathrm{GR} / \mathrm{DE})$ & 372 & 4 & & \\
\hline & & ESUN & 8 & 2 & & \\
\hline & & ESHS & 10 & 1 & & \\
\hline & \multirow{5}{*}{$\mathrm{EV}(48)$} & EVHS & 2815 & 29 & & \\
\hline & & EVUN & 22 & 7 & & \\
\hline & & EVSU & 818 & 6 & & \\
\hline & & EVOM & 5191 & 5 & & \\
\hline & & EVDE & 86 & 1 & & \\
\hline \multirow{4}{*}{ 内栖(14) } & IS(1) & ISHS & 244 & 1 & \multirow{4}{*}{-0.276} & \multirow{4}{*}{25.9} \\
\hline & \multirow{3}{*}{$\operatorname{IV}(13)$} & IVHS & 3649 & 8 & & \\
\hline & & IVSU & 622 & 4 & & \\
\hline & & IVDE & 262 & 1 & & \\
\hline
\end{tabular}

a) 基于野外采样数据, 见网络版附表 2

表 5 澄江动物群取食方式类型统计表 ${ }^{a)}$

\begin{tabular}{|c|c|c|c|c|c|}
\hline \multirow{2}{*}{ 取食策略(缩写) } & \multicolumn{2}{|c|}{ 基于文献统计的数据 } & \multicolumn{3}{|c|}{ 野外采集统计的个体丰度数据 } \\
\hline & 物种数 & 物种比率(\%) & 物种数 & 物种比率(\%) & 个体丰度 $(\%)$ \\
\hline 捕食/食腐者(HS) & 70 & 31.1 & 49 & 43 & 40.4 \\
\hline 滤食者(SU) & 80 & 35.6 & 42 & 36.8 & 26.1 \\
\hline 食泥者(DE) & 7 & 3.1 & 2 & 1.8 & 1.9 \\
\hline 杂食者(OM) & 4 & 1.8 & 5 & 4.4 & 28.2 \\
\hline 不能确定(UN) & 64 & 28.4 & 16 & 14 & 3.4 \\
\hline
\end{tabular}

a) 基于发表的数据和野外采样数据的对比

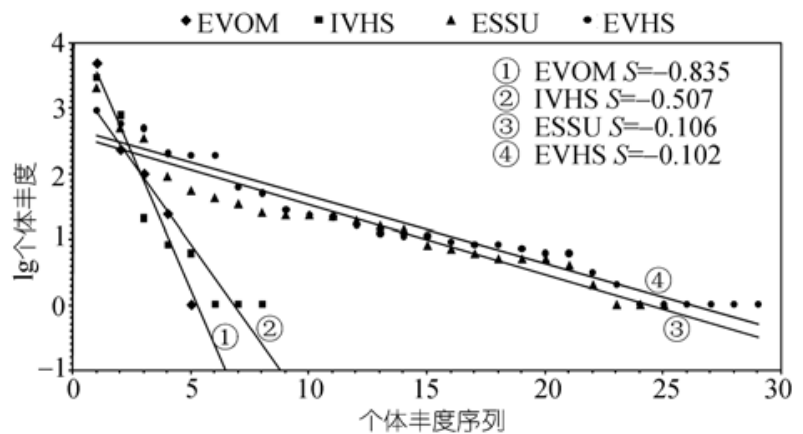

图 13 物种最丰富的四类生态功能群的 Whittaker 点图

图中标注出每一类点集形成的线性回归线和斜率值

层区域, 其被捕获而被埋藏的可能性就低. 而底栖物 种或处于水层下部的物种在风暴引起的絮状沉积物 快速沉降范围内, 可以有效的对其捕获. 这可能是澄
江动物群以底栖物种个体数量高的主要原因之一, 当然并不排除化石的这种分析结果为原始群落特征 的反映.

\section{5 讨论}

\section{1 埋藏偏差对群落分析的影响}

古群落的研究中, 埋藏偏差对化石组合的影响 是不容忽视的, 如生物体的腐烂、生物扰动、搬动、 时间均衡、生物矿化、后期改造等, 地层中保存的化 石组合通常是由具生物矿化壳体或骨骼的物种组成, 这与最初的原始群落面貌相差甚远. 即使是特异埋 藏软躯体动物化石群, 如澄江动物群、布尔吉斯页岩 动物群, 其软躯体化石保存程度与区域埋藏相也是 


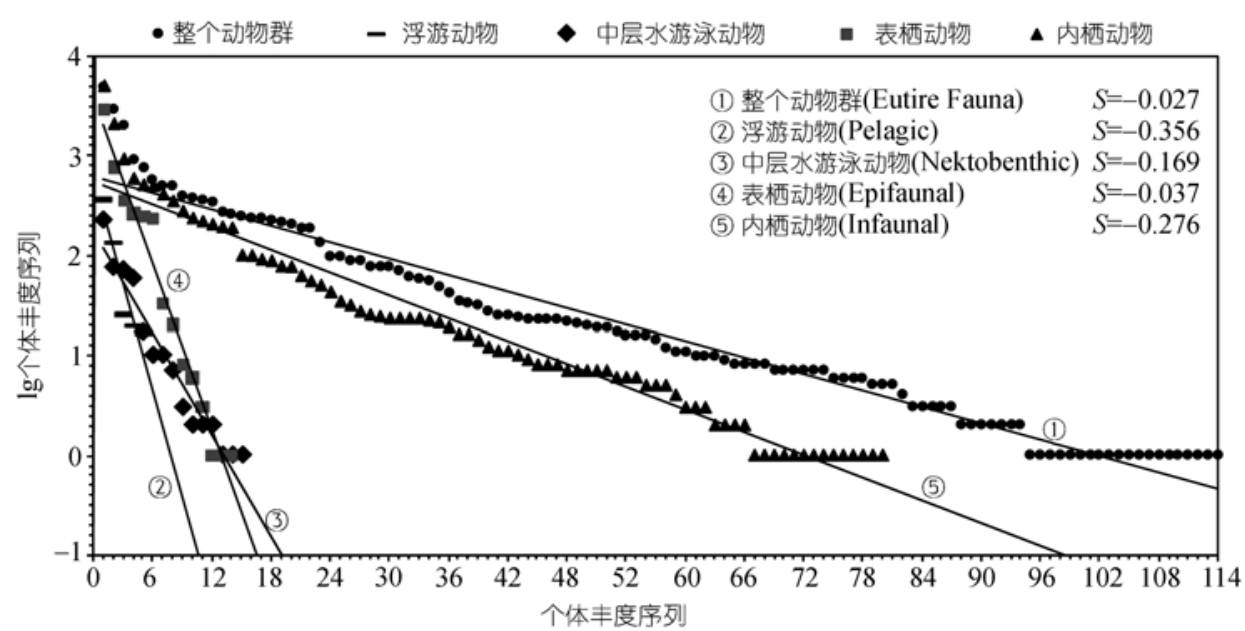

图 14 水体空间四类生活型和整个群落的 Whittaker 点图

图中标注出每一类点集形成的线性回归线和斜率值

密切相关的 ${ }^{[9,13]}$, 在澄江-海口-安宁地区产出的化石 多数都保存有非生物矿化的软组织器官, 其产出的 层位生物扰动遗迹弱, 化石多为软躯体个体的单属 种聚集或多属种富集保存型式 ${ }^{[9,35]}$, 埋藏微相和化石 保存的研究指示该地区的化石为原地或近栖息地埋 藏 ${ }^{[9,11,12]}$, 其沉积围岩形成于风暴引起的悬浮泥质絮 状物快速沉积 ${ }^{[9,11,12,18,19]}$. 因此生物腐烂、生物扰动、 搬运对这一地区的化石群落产生偏差较少. 由于澄 江动物群特殊的埋藏模式, 生物化石多为活着时被 保存, 那么生物本身因素可能会对被埋藏下的生物 组合产生一定的篎选. 因为具有不同生活方式和不 同身体大小的物种对泥质沉积事件的反映是不一致 的, 也就是说生物逃逸能力差异的存在可能对最终 保存下来的生物组合产生影响. 如底表和底内生物 生活在海洋底部沉积物表面或内部, 根据Brett等 ${ }^{[36]}$ 对底栖固着生活的生物研究(如海绵)显示, 当快速沉 积事件发生后, 毫无疑问, 无论风暴引起的絮状物沉 积有多慢, 对于这种固着的几乎无逃逸能力的生物, 也只有等待被埋藏. 内栖或表栖活动的生物, 在事件 沉积发生时具有一定的逃逸能力, 澄江动物群里大 量的底栖动物的存在, 说明多数底栖生物在沉积事 件发生时并不能有效的逃逸. 另外值得一提的是, 澄 江生物群的生物个体都比较小, 大多小于 $2 \mathrm{~cm}$. 个体 较小、活动能力差的底栖生物逃逸能力相对较差, 这 可能是众多底栖动物化石被保存在泥岩中的一个重 要原因. 浮游生物种类和个体数量在事件层泥岩内
保存相对少，可能与其通常生活在水体上层有关，风 暴引起的絮状泥质沉积物并不能有效到达其生活区 域对其有效的捕获，但当浮游生物捕食或其他生活 习性而进入风暴沉积水体区域, 或由于其他特殊外 界原因, 如缺氧等. 就可能会保存在事件泥岩层内.

\section{2 不同区域化石组合的对比}

澄江动物群生物多样性具有明显的空间变 化 ${ }^{[9,13,37]}$. 在武定地区, 帽天山页岩段以发育大量生 物扰动为特征, 物种分异度最低, 目前只有 26 个物 种被发现 ${ }^{[13]}$. 节肢动物主控这一地区性群落, 个体数 量占 $47.1 \%$, 其后是软舌螺类 $(33.7 \%)$ 和腕足动物 (18.7\%). 最丰富的物种是软舌螺Ambrolinevitus ventricosus (29.7\%), 其后是节肢动物Wutingaspis tingi $(24.2 \%)$ 和腕足动物Diandongia pista $(18.3 \%)^{[9]}$. 在马 龙-曲靖地区, 沉积物具有受水体扰动和搬运的特征, 具有中等物种分异度 $(55 \text { 个物种 })^{[13]}$, 主控门类是节 肢动物, 个体丰度达到 $90 \%$. 最丰富的物种是节肢 动物 Kunmingella douvillei 和 Wutingaspis malungensis ${ }^{[9]}$. 澄江-海口-安宁一带帽天山页岩段主要以背 景泥岩层和事件泥岩层相互偶合式叠加为特征 ${ }^{[12]}$, 因而受埋藏因素影响最少, 生物分异度达到最高(210 个物种 $)^{[13]}$, 被认为有最大潜力反映澄江动物群原始 群落特征 ${ }^{[12,39]}$.

目前澄江-海口-安宁一带较为详细的古生态定 量分析主要是来自安宁山口村和海口马房村两个剖 
面. Dornbos等 ${ }^{[38]}$ 对山口村的化石采集的定量研究指 出曳鳃动物主控这一地区的生物群落, 5 个优势种其 中 3 个是曳鳃动物(Paraselkirkia, Sicyophorus, Maotianshania), 另外两个分别是腕足动物 (Heliomedusa) 和节肢动物(Leanchoilia), 5 个优势种个体丰度占到 59\%. 而我们对海口马房村剖面详细研究结果表明, 节肢动物主控这一地区 ${ }^{[12,39]}$, 五个优势种有两个节 肢动物 (Kunmingella, Leanchoilia), 两个曳鳃动物 (Cricocosmia, Mafangscolex) 和一个腕足动物 (Diandongia) 占到总个体数量的 $71.4 \%$. 这种结果的 差异可能是由于 Dornbos等 ${ }^{[38]}$ 研究的化石数据主要 是来自己经收藏在实验室内的化石标本整理的缘故. 因为最初的化石采集并不是针对古生态学研究为目 的的采集, 大量常见的和保存不好的化石并没有充 分采集, 而我们在海口马房村剖面所获得的数据是 在野外定量统计获得的.

从大区域上来看, 澄江动物群分异度空间差异 主要受埋藏因素控制. 然而, 在化石保存的核心相区, 具有相同埋藏相的澄江、海口、安宁三个地区不同剖 面的物种分异度、物种组成和个体丰度在空间上存在 明显的变化 ${ }^{[2,9,12,37]}$, 这种变化除埋藏因素外, 生态因 素也是一个极其重要的控制因素 ${ }^{[13]}$, 物种分布可能 受到环境和地形等小生境影响. 因而, 对核心埋藏相 区采用系统的化石采集, 定量分析其化石群落定能 够概略性反映出寒武纪早期重要的海洋浅海古群落 信息.

\section{3 与布尔吉斯页岩动物群的对比}

寒武纪早期中国澄江动物群和寒武纪中期加拿 大布尔吉斯页岩动物群是两个重要的特异埋藏化石 群, 因而对比两个动物群的群落特征, 将对揭示寒武 纪生物演化过程具有重要意义. 从生物门类构成来 看, 两个动物群拥有的动物门类大多数相同. 节肢动 物无论是物种分异度还是个体丰度在两个动物群都 占有主控地位. 两个动物群中海绵动物门、曳鰓动物 门、腕足动物门具有较高的物种分异度. 脊索动物在 布尔吉斯页岩动物群物种数量和个体丰度都非常稀 少 ${ }^{[40 \sim 42]}$, 而在澄江动物群具有相对高的个体丰度和 物种分异度, 已经报道的物种数有 10 种 ${ }^{[24,43 \sim 46]}$. 与 布尔吉斯页岩动物群相比, 半索动物和棘皮动物在 澄江动物群保存稀少, 多数种类其门类属性争议较 $大^{[23,44,46-51]}$. 迄今为止, 多毛类在澄江动物群里一直
没有确切的报道，依据一块保存差的标本命名的 Maotianchaeta fuxianella 被报道作为可疑的多毛类 ${ }^{[2]}$. 相反, 在布尔吉斯页岩动物群里, 半索动物、棘皮动 物和多毛类具有明显高的物种分异度和个体丰度 ${ }^{[20]}$.

从生态功能群组成来看, 两个动物群具有相同 的生态功能群组成模式, 都以底栖动物为主, 并且包 括少数浮游动物, 水体生态空间内的物种分异度相 似, 滤食性生物的分异度最高, 捕食或食腐生物分异 度高于食沉积物的生物. 与Caron等 ${ }^{[21]}$ 的布尔吉斯页 岩动物群物种数据对比, 澄江动物群和布尔吉斯页 岩动物群的底内生物(IV和IS)分别为 $11.9 \%$ 和 $11 \%$; 底表生物(ES和EV)在各动物群都占有最大比例, 澄 江动物群有 $63 \%$, 布尔吉斯页岩动物群有 $48.3 \%$; 游 泳生物(NK)在布尔吉斯页岩动物群有 $10.9 \%$, 澄江动 物群有 $11.5 \%$. 浮游生物在整个生态群中也占有一定 比例, 布尔吉斯页岩动物群有 $10 \%$, 澄江动物群有 $5.3 \%$. 藻类等蓝细菌(EAPP) 在布尔吉斯页岩动物群 物种数量占 $9 \%$, 澄汇动物群只占 $1 \%$; 在两个动物群 中物种主要以滤食者为主, 在澄江动物群的物种占 有 $35.3 \%$, 在布尔吉斯动物群的物种占有 $35.8 \%$. 捕 食或食腐者次之, 澄江动物群有 $31.3 \%$ 的物种为捕食 或食腐者, 布尔吉斯页岩动物群有 $27.7 \%$ 物种为捕食 或食腐者. 拥有物种数量最多的生态功能群在两个 动物群都是表栖固着滤食类, 布尔吉斯页岩动物群 有 37 种，占 $21 \%$; 澄江动物群有 42 种，占 $18.5 \%$. 其 次是表栖活动捕食或食腐者(EVHS), 布尔吉斯页岩 动物群有 17 种, 占 $10 \%$; 澄江动物群有 37 种, 占 $16.3 \%$. 值得注意的是, 澄江动物群中食沉积物者的 分异度明显低于布尔吉斯页岩动物群, 澄江动物群 有 7 种, 占 $3.1 \%$; 布尔吉斯页岩生物群有 32 种, 占 $20 \%$.

Powell等 ${ }^{[52]}$ 对布尔吉斯页岩动物群埋藏环境研 究认为, 布尔吉斯页岩动物群可能还是以微生物为 基础构建起来的食物网系统，仍然相似于寒武纪底 质革命 ${ }^{[53,54]}$ 之前的食物网形式. 这个结论存在很大 争议 ${ }^{[21,55]}, \mathrm{Caron}^{[56]}$ 和Conway ${ }^{[20]}$ 对布尔吉斯页岩动物 群古群落研究认为, 对布尔吉斯页岩动物群古群落 食物网已相对复杂，已经演化具有现在海洋生物群 落食物网特征. 我们对澄江动物群不同取食方式的 生物的分析研究表明, 寒武纪食物网明显不同于前 寒武纪微生物席为基础的食物网结构, 已发展成类 似于现在浅海底栖群落的食物构型。澄江动物群和 
布尔吉斯页岩动物群里的化石肠道保存物、取食器官 形态功能、粪化石成分、捕食迹等分析, 都指示了寒 武纪多层营养级类似现代金字塔构架的食物网的存 在. 最近, Dunne等 ${ }^{[57]}$ 通过拓扑结构分析, 对比了澄 江动物群和布尔吉斯页岩动物群食物网构成, 也认 为尽管这两个动物群所体现出的食物网拓扑结构相 对简单, 但已经十分类似于现在海洋的食物网结构.

\section{6 结论}

通过物种和个体丰度数据定量分析, 揭示了节 肢动物无论是物种丰度还是个体丰度都主控澄江动 物群, 曳鳃动物和腕足动物也具有相对高的个体丰 度. 节肢动物 Kunmingella douvillei (26.2\%), 曳鳃动 物 Cricocosmia jinningensis(15.4\%) 和腕足动物
Diandongia pista $(11 \%)$ 是个体丰度最高的物种. 整个 澄江动物群主要以底栖动物为主, 表栖活动杂食类 $(\mathrm{EVOM}) 、$ 表栖活动捕食/食腐类 $(\mathrm{EVHS})$ 、底内活动 捕食/食腐类(IVHS)和表栖固着滤食类(ESSU)是个体 丰度最高的四个生态功能群. 生态功能群的分析揭 示了寒武纪澄江动物群与现代浅海生物群落具有相 同的群落构成, 物种生存空间扩大到沉积物内到上 层水体, 大多数物种具有捕食、食腐和滤食取食策略, 预示着由多层次营养级结构构成的金字塔式的食物 网在寒武纪早期已经形成. 以上分析表明, 澄江动物 群由多门类具有不同复杂生态功能的物种组成, 揭 示了寒武纪早期物种多样的复杂性浅海生态系统. 以上结论为探索寒武纪后生动物多样性形成、寒武纪 生态空间扩张以及寒武纪早期类似现代海洋生态系 统建立提供了定量的化石证据. 文章初稿的有益建议; 同时也感谢杨志、马房村民和地方政府的支持和野外采集化石所给予的帮助.

\section{参考文献}

1 Zhu M Y, Babcock L E, Peng S C. Advances in Cambrian stratigraphy and paleontology: Integrating correlation techniques, paleobiology, taphonomy and paleoenvironmental reconstruction. Palaeoword, 2006, 15: 217-222

2 陈均远. 动物世界的黎明. 南京: 江苏科技出版社, 2004. 366

3 Hou X G, Aldridge R J, Bergström J, et al. The Cambrian Fossils of Chengjiang, China: The Flowering of Early Animal Life. Oxford: Blackwell Publishing Company, 2004. 233

4 Hou X G, Siveter D J, Aldridge R J, et al. Collective behavior in an Early Cambrian arthropod. Science, 2008, $322: 224$

5 Hu S X, Steiner M, Zhu M Y, et al. Diverse pelagic predators from the Chengjiang Lagerstätte and the establishment of modern-style pelagic ecosystems in the Early Cambrian. Palaeogeogr Palaeoclimat Palaeoecol, 2007, 254: 307-316

6 Shu D G. Cambrian explosion: Birth of tree of animals. Gondwana Res, 2008, 14: 219-240

7 张文堂, 侯先光. Naraoia 在亚洲大陆的发现. 古生物学报, 1985, 24: 591-595

8 Zhu M Y, LI G X, Zhang J M, et al. Early Cambrian stratigrapgy of East Yunnan, Southwestern China: A synthesis. Acta Palaeontol Sin, 2001, 40(Suppl): 4-39

9 Hu S X. Taphonomy and palaeoecology of the Early Cambrian Chengjiang biota from eastern Yunnan, China. Berl Paläobiolog Abhand, 2005, 7: $1-197$

10 Zhang X L, Shu D, Han J, et al. New sites of Chengjiang fossils: Crucial windows on the Cambrian explosion. J Geol Soc, 2001, 158: 211218

11 Zhu M Y, Zhang J M, Li G X. Sedimentary environments of the Early Cambrian Chengjiang biota: Sedimentology of the Yu'anshan Formation in Chengjiang County, Eastern Yunnan. Acta Palaeontol Sin, 2001, 40(Suppl): 80-105

12 Zhao F C, Caron J B, Hu S X, et al. Quantitative analysis of Taphofacies and Paleocommunities in the early Cambrian Chengjiang Lagerstätte. Palaios, 2009, 24: 826-839

13 Zhao F C, Hu S X, Caron J B, et al. Spatial variation in species diversity and composition of the early Cambrian Maotianshan Shale Biota: Taphonomic versus Ecological controls. In: Abstracts of the International Conference on the Cambrian Explosion. 2009, http://www. burgess-shale.info/abstract/f-zhao

14 罗惠麟, 蒋志文, 武希彻, 等. 云南东部震旦系-寒武系界线. 昆明: 云南人民出版社, 1982. 265

15 Erdtmann B D, Steiner M. Special observation concerning the Sinian-Cambrian transition and its stratigraphic implications on the central 
and SW Yangtze Plateform, China. Palaeoworld, 2001, 13: 52-65

16 Jin Y G, Wang H Y, Wang W. Palaeoecological aspect of branchiopods from Chiungchussu Formation of Early Cambrian age, Eastern Yunan, China. Palaeoecol China, 1991, 1: 25-47

17 蒲心纯, 周浩达, 王熙林, 等. 中国南方寒武纪岩相古地理与成矿作用. 北京: 地质出版社, 1992. 191

18 孙枢, 范德廉, 陈海泓, 等. 中国地台区张裂盆地沉积. 沉积学报, 1987, 5: 6-18

19 孙枢, 陈海泓. 滇东下寒武统的风暴沉积. 见: 中国石油学会石油地质委员会, 编. 碎屑岩沉积相研究. 北京: 石油工业出版社, 1988. 357-363

20 Conway M S. The community structure of the Middle Cambrian phyllopod bed (Burgess shale). Palaeontology, 1986, 29: 423-467

21 Caron J B, Jackson D A. Paleoecology of the greater phyllopod bed community, Burgess shale. Palaeogeogr Palaeoclimat Palaeoecol, 2008, 258: $222-256$

22 Shu D G, Conway Morris S, Han J, et al. Primitive deuterostomes from the Chengjiang Lagerstätte (Lower Cambrian, China). Nature, 2001, 414: $419-424$

23 Shu D G, Conway M S, Han J, et al. Ancestral echonoderms from the Chengjiang deposits of China. Nature, 2004, 430: 422-427

24 Shu D G, Luo H L, Conway M S, et al. Lower Cambrian vertebrates from south China. Nature, 1999, 402: 42-46

25 舒德干. 脊椎动物实证起源. 科学通报, 2003, 48: 541-550

26 Leslie S A, Babcock L E, Zhang W T. Community composition and taphonomic overprint of the Chengjiang biota (Early Cambrian, China). In: Repetski J, ed. Sixth North American Paleontological Convention Abstract of Papers. Paleontol Soc Spec Publ, 1996, 8: 237

27 舒德干. 再论古虫动物门. 科学通报, 2005, 50: 2114-2126

28 Hou X G, Ramsköld L, Bergström J. Composition and preservation of the Chengjiang fauna-A lower Cambrian soft-bodied biota. Zool Script, 1991, 20: 395-411

29 Chen J Y, Huang D Y, Li C W. An Early Cambrian craniate-like chordate. Nature, 1999, 402: 518 - 522

30 Chen J Y, Huang D Y, Peng Q Q, et al. The first tunicate from the Early Cambrian of South China. Proc Natl Acad Sci USA, 2003, 100: $8314-8318$

31 Shu D G, Chen L, Han J, et al. An Early Cambrian tunicate from China. Nature, 2001, 411: 472-473

32 Shu D G, Conway M S, Han J, et al. Lower Cambrian vendobionts from China and Early diploblast evolution. Science, 2006, 312: 731734

33 Magurran A E. Measuring Biological Diversity. Oxford: Blackwell Publishing Company, 2004. 256

34 Whittaker R H. Dominance and diversity in land plant communities. Science, 1965, 147: 250-260

35 韩建, 舒德干, 张志飞, 等. 早寒武世澄江化石库软躯体化石富集层研究初探. 科学通报, 2006, 51: 565一 574

36 Brett C E, Seilacher A. Fossil Lagerstätten: A taphonomic consequence of single-event sedimentation. In: Einsele G, Ricken W, Seilacher A, eds. Cycles and Single-events in Stratigraphy. New York: Springer-Verlag, 1991. 283-297

37 Steiner M, Zhu M Y, Zhao Y L, et al. Lower Cambrian Burgess shale-type fossil associations of South China. Palaeogeogr Palaeoclimat Palaeoecol, 2005, 220: 129-152

38 Dornbos S Q, Chen J Y. Community palaeoecology of the early Cambrian Maotianshan Shale biota: Ecological dominance of priapulid worms. Palaeogeogr Palaeoclimat Palaeoecol, 2008, 258: 200-212

39 赵方臣, 朱茂炎. 云南早寒武世澄江化石库中两种埋藏相的化石定量分析. 古生物学报, 2007, 46: 75一86

40 Simonetta A M, Emilio I. New animals from the Burgess Shale (Middle Cambrian) and their possible significance for the understanding of the Bilateria. Boll Zool, 1993, 60: 97-107

41 Briggs D E G, Erwin D H, Collier F J. The Fossils of the Burgess Shale. Washington D C: Smithsonian Institution Press, 1994. 238

42 Janvier P. Vertebrate characters and the Cambrian vertebrates. Compt Rend Palevol, 2003, 2: 523-531

43 Chen J Y, Dzik J, Edgecombe G D, et al. A possible Early Cambrian chordate. Nature, 1995, 377: 720 - 722

44 Chen J Y, Zhou G Q. Biology of the Chengjiang fauna. Bull Natl Mus Nat Sci-Taichung, 1997, 10: 11—106

45 Shu D G, Conway M S, Han J, et al. Head and backbone of the Early Cambrian vertebrate Haikouichthys. Nature, 2003, 421: 526-529

46 Mallatt J, Chen J Y. Fossil sister group of Craniates: Predicted and found. J Morphol, 2003, 258: 1-31

47 Shu D G, Conway M S, Zhang X L. A Pikaia-like chordate from the Lower Cambrian of China. Nature, 1996, 384: 157-158

48 Shu D G, Conway M S, Zhang Z F, et al. A new species of Yunnanozoan with implications for deuterostome evolution. Science, 2003, 299: $1380-1384$

49 Shu D G, Conway M S. Response to comment on “a new species of Yunnanozoon with implications for deuterostome evolution”. Science, 2003, 300: 1372 
50 Shu D G, Gonway M S, Zhang Z F, et al. The earliest history of the deuterostomes: The importance of the Chengjiang Fossil-Lagerstätte. Proc R Soc B, 2010, 277: 165-174

51 Shu D G, Zhang X L, Chen L. Reinterpretation of Yunnanozoon as the earliest known hemichordate. Nature, 1996, 380: 428 - 430

52 Powell W G, Johnston P A, Collom C J. Geochemical evidence for oxygenated bottom waters during deposition of fossiliferous strata of the Burgess Shale Formation. Palaeogeogr Palaeoclimat Palaeoecol, 2003, 201: 249-268

53 Seilacher A. Biomat-related lifestyles in the Precambrian. Palaios, 1999, 14: 86-93

54 Bottjer D J, Hagadorn J W, Dornbos S Q. The Cambrian substrate revolution. GSA Today, 2000, 10: 1—7

55 Dornbos S Q, Bottjer D J, Chen J Y. Paleoecology of benthic metazoans in the Early Cambrian Maotianshan shale biota and the Middle Cambrian Burgess shale biota: Evidence for the Cambrian substrate revolution. Palaeogeogr Palaeoclimat Palaeoecol, 2005, 220: 47—67

56 Caron J B. Taphonomy and community analysis of the Middle Cambrian Greater Phyllopod Bed, Burgess Shale. Doctoral Dissertation. Toronto: University of Toronto, 2004

57 Dunne J A, Williams R J, Martinez N D, et al. Compilation and network analyses of Cambrian food webs. PLoS Biol, 2008, 6: e102. doi: 10.1371/journal.pbio.0060102 


\section{附表 1 澄江生物群物种门类和生态功能群分类}

\begin{tabular}{|c|c|c|c|}
\hline 生物门 & 生活方式 & & 物种 \\
\hline \multirow{28}{*}{ PORIFERA } & \multirow{28}{*}{ ESSU } & \multirow{28}{*}{28} & Allantospongia mica Rigby \& Hou, 1995 \\
\hline & & & Choia carteri Walcott, 1920 \\
\hline & & & $\begin{array}{l}\text { Choia Hindei Dawson, 1896(=Choia xiaolantianensis Hou, Bergström, Wang, Feng \& Chen } \\
\text { 1999) }\end{array}$ \\
\hline & & & Choia ridleyi Walcott, 1920 \\
\hline & & & Choia sp. Luo et al. 1999 \\
\hline & & & Choia utahensis Walcott, 1921 \\
\hline & & & Choiaella radiata Rigby \& Hou, 1995 \\
\hline & & & Cystospongia globose Wu, 2004(=Crumillespongia sp. nov Chen et al. 1996) \\
\hline & & & Halichondrites elissa Walcott, 1920(=Halichondrites sp. nov. Chen et al. 1997) \\
\hline & & & Hamptonia chengjiangensis $\mathrm{Wu}, 2004$ \\
\hline & & & Hazelia sp. nov. Chen et al. 1996(=? Hazelia palmata Walcott, 1920) \\
\hline & & & Hyalocinica sp. Wu, 2004 \\
\hline & & & Ischnspongia dendriticagen $\mathrm{Wu}, 2004$ \\
\hline & & & Leptomitella confusa Chen, Hou \& Lu, 1989 \\
\hline & & & Leptomitella conica Chen, Hou \& Lu, 1989 \\
\hline & & & Leptomitus teretiusculus Chen, Hou \& Lu, 1989 \\
\hline & & & Leptomitus zitteli Walcott, 1886 \\
\hline & & & Paraleptomitella dictyodroma Chen, Hou \& Lu, 1989 \\
\hline & & & Paraleptomitella globula Chen, Hou \& Lu, 1989 \\
\hline & & & Protospongia sp. Wu, 2004 \\
\hline & & & Ptilispongia maotianshanensis Wu, 2004 \\
\hline & & & Quadrolaminiella crassa Chen, Hou \& Li, 1990 \\
\hline & & & Quadrolaminiella diagonalis Chen, Hou \& Li, 1990 \\
\hline & & & Saetaspongia densa Mehl \& Reitner in Steiner et al., 1993 \\
\hline & & & Takakkawia sp. nov. Chen et al. 1996 \\
\hline & & & Triticispongia diagonata Mehl \& Reitner in Steiner et al., 1993 \\
\hline & & & Valospongia gigantis Rigby, 1983 \\
\hline & & & Wapkia grandis Walcott, 1920 \\
\hline CHANCELLORIIDS & $\mathrm{ES}(\mathrm{SU} / \mathrm{HS})$ & 1 & $\begin{array}{l}\text { Allonina phrixothrix Bengtson \& Hou, 2001(=allonina junyuan Janussen, Steiner et Zhu, } \\
2002=\text { Chancelloria eros in Chen et al. 2002) }\end{array}$ \\
\hline \multirow{7}{*}{ CNIDARIA } & $\mathrm{ES}(\mathrm{HS} / \mathrm{SU})$ & 1 & Priscapennamarina agnusta Zhang \& Babcock, 2001 \\
\hline & & & Archisaccophyllia kunmingensis Hou et al., 2005 \\
\hline & ESSU & 2 & $\begin{array}{l}\text { Archotuba conoidalis Hou, Bergström, Wang, Feng \& Chen, 1999(=? Selkirkia elongate Luo \& } \\
\text { Hu, } 1999=\text { Cambrorhytium sp. in Chen et al. 1996) }\end{array}$ \\
\hline & EVHS & 1 & Cambrohydra ercaia $\mathrm{Hu}, 2005$ \\
\hline & IVHS & 2 & Conicula striata Luo \& Hu, 1999 \\
\hline & IVHS & 2 & Xiangguangia sinica Chen \& Erdtmann, 1991 \\
\hline & UNUN & 1 & Chengjiangopenna wangii Shu and Conway Morris, 2006 \\
\hline \multirow{7}{*}{ CTENOPHORA } & & & Batofasciculus ramificans Hou, Bergström, Wang, Feng \& Chen, 1999 \\
\hline & & & Ctenophora New genus in Chen, 2004 \\
\hline & PEHS & 5 & Maotianoascus octonarius Chen \& Zhou, 1997 \\
\hline & & & Trigoides aclis Luo \& Hu, 1999 \\
\hline & & & Yunnanoascus Haikouensis Hu, 2005 \\
\hline & PEHS? & 1 & *Sinoascus papillatus Chen \& Zhou, 1997 \\
\hline & UNUN & 1 & Stromatoveris psygmoglena Shu et Conway Morris, 2006 \\
\hline \multirow{3}{*}{ PRIAPULIDA } & $\mathrm{EV}(\mathrm{DE} / \mathrm{HS})$ & 1 & *Yunnanpriapulus halteroformis Huang, Vannier \& Chen, 2004 \\
\hline & EVUN & 1 & Tylotites petioaris Luo \& Hu, 1999 \\
\hline & ISHS & 1 & $\begin{array}{l}\text { Selkirkia sinica Luo \& Hu, 1999(?= paraselkirkia jinningensis } \text { Hou, Bergström, Wang, Feng \& } \\
\text { Chen, 1999) }\end{array}$ \\
\hline
\end{tabular}




\begin{tabular}{|c|c|c|c|}
\hline 生物门 & 生活方式 & & 物种 \\
\hline \multirow{16}{*}{ PRIAPULIDA } & \multirow{3}{*}{ IVDE } & \multirow{3}{*}{3} & Acosmia maotiania Chen \& Zhou, 1997 \\
\hline & & & $\begin{array}{l}\text { Sicyphorus rara Luo \& Hu, 1999(=protopriapulites haikouensis } \text { Hou, Bergström, Wang, Feng } \\
\text { \& Chen, 1999=Palaeopriapulites parvus } \text { Hou, Bergström, Wang, Feng \& Chen, 1999) }\end{array}$ \\
\hline & & & Palaeopriapulites parvus Hou, Bergström, Wang, Feng \& Chen, 1999 \\
\hline & \multirow{10}{*}{ IVHS } & \multirow{10}{*}{10} & Anningvermis multispinosa Huang, Vannier \& Chen, 2004 \\
\hline & & & Corynetis brevis Luo \& Hu, 1999 \\
\hline & & & Paratubiluchus bicaudatus Han, Shu, Zhang \& Liu, 2004 \\
\hline & & & Tabelliscolex hexagonus Han, Zhang et Shu, 2003 \\
\hline & & & Tabelliscolex chengjiangensis Han, Lui et al, 2007 \\
\hline & & & Xiaoheiqingella peculiatis $\mathrm{Hu}, 2002$ \\
\hline & & & Cricocosmia jinningensis Hou \& Sun, 1988(=Spinovermis yunnanicus Yang, 1999) \\
\hline & & & Omnidens amplus Hou et al., 2006 \\
\hline & & & Maotianshania cylindrica Sun \& Hou, 1987 \\
\hline & & & Mafangscolex sinensis Hou \& Sun, 1988 \\
\hline & IVHS? & 1 & *Xishania longiusula $\mathrm{Hu}, 2002$ \\
\hline & \multirow{2}{*}{ UNUN } & \multirow{2}{*}{2} & *Lagenula striolata Luo \& Hu, 1999 \\
\hline & & & *Sandaokania latinodosa Luo \& Hu, 1999 \\
\hline \multirow{2}{*}{ SIPUNCULAN } & \multirow{2}{*}{ IVDE? } & \multirow{2}{*}{2} & Archaeogolfingia caudata Huang, Chen, Vannier \& Salinas, 2004 \\
\hline & & & Cambrosiphunculus tentaculatus Huang, Chen, Vannier \& Salinas, 2004 \\
\hline ANNELIDA & UNUN & 1 & ?Maotianchaeta fuxianella Chen, 2004 \\
\hline \multirow{8}{*}{ HYOLITHA } & \multirow{8}{*}{$\mathrm{ES}(\mathrm{GR} / \mathrm{DE})$} & \multirow{8}{*}{8} & Ambrolinevitus ventricosus Qian, 1978 \\
\hline & & & Ambrolinevitus platypluteus Qian, 1978 \\
\hline & & & Ambrolinevitus meishucunensis Jiang, 1994 \\
\hline & & & Ambrolinevitus maximus Jiang, 1982 \\
\hline & & & $\begin{array}{l}\text { Glossolites magnus Luo \& Hu, } 1999 \text { (Burithes yunnanensis Hou, Bergström, Wang, Feng \& } \\
\text { Chen, 1999) }\end{array}$ \\
\hline & & & Linevitus billingsi Walcott, 1886 \\
\hline & & & Linevitus flabellaris Qian, 1978 \\
\hline & & & Linevitus opimus Yu, 1974 \\
\hline \multirow{9}{*}{ BRACHIOPODA } & \multirow{4}{*}{ ESSU } & \multirow{4}{*}{4} & Diandongia pista Rong, 1974 (Obolus in Zhang et al. 2001) \\
\hline & & & Heliomedusa orienta Sun \& Hou, 1987 \\
\hline & & & $\begin{array}{l}\text { Longtancunella chengjiangensis Hou, Bergström, Wang, Feng \& Chen, 1999(=? Paleolingula } \\
\text { Shu et al. 1993) }\end{array}$ \\
\hline & & & Undescribed brachiopod in $\mathrm{Hu}, 2005$ \\
\hline & \multirow{3}{*}{ IVSU } & \multirow{3}{*}{3} & Lingulella chengjiangensis Jin, Hou \& Wang, 1993 \\
\hline & & & Lingulellotreta malongensis Rong, 1974(=Lingulepis malongensis in Chen et al. 1996) \\
\hline & & & Wangyuia yunnanensis Jin, 2004 \\
\hline & \multirow{2}{*}{ ES?SU } & \multirow{2}{*}{2} & *Xianshanella haikouensis Zhang \& Han, 2004 \\
\hline & & & Kutorgina chengjiangensis Zhang et al., 2007 \\
\hline PHORONIDA & IVHS & 1 & Eophoronis chengjiangensis Chen, 2004(=Iotuba chengjiangensis chen and zhou 1997) \\
\hline CHAETOGNATHA & PEHS & 1 & Protosagita spinosa $\mathrm{Hu}, 2002(=$ Eognathacantha ercainella Chen \& Huang, 2002) \\
\hline \multirow{9}{*}{ LOBOPODIA } & \multirow{9}{*}{ EVHS } & \multirow{9}{*}{12} & Aysheaia sp.Hu, 2005 \\
\hline & & & Cardiodictyon catenulum Hou Ramsköld \& Bergström, 1991 \\
\hline & & & Hallucigenia fortis Hou \& Bergström, 1995 \\
\hline & & & Facivermis yunnanicus Hou \& Chen, 1989 \\
\hline & & & Luolishania longicruris Hou \& Chen, 1989 \\
\hline & & & Megadictyon haikouensis Luo \& Hu, 1999 \\
\hline & & & Microdictyon sinicum Chen, Hou \& Lu, 1989 \\
\hline & & & *Miraluolishania haikouensis Liu, Shu, Han \& Zhang, 2004 \\
\hline & & & Onychodictyon ferox Hou Ramsköld \& Bergström, 1991 \\
\hline
\end{tabular}




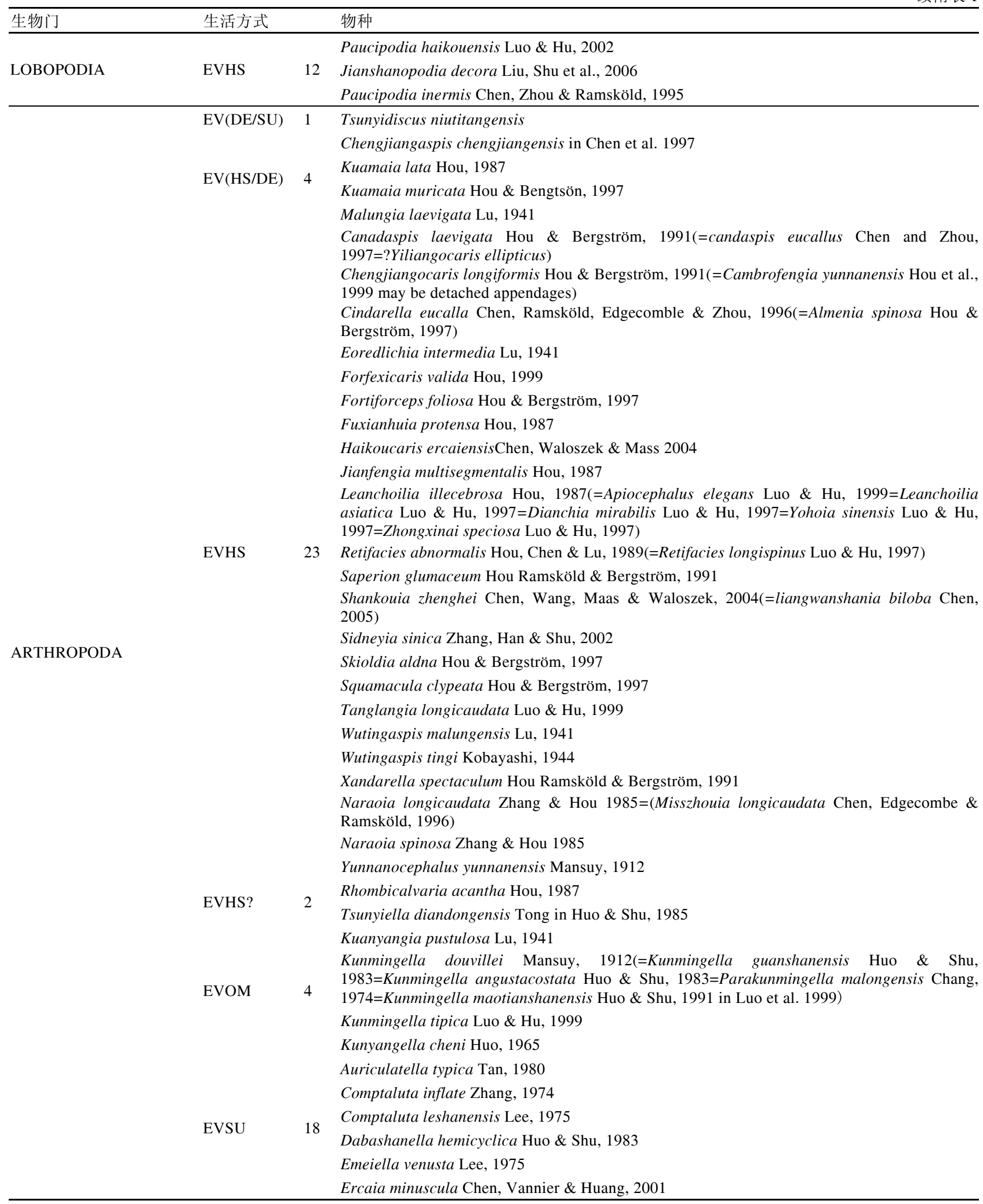




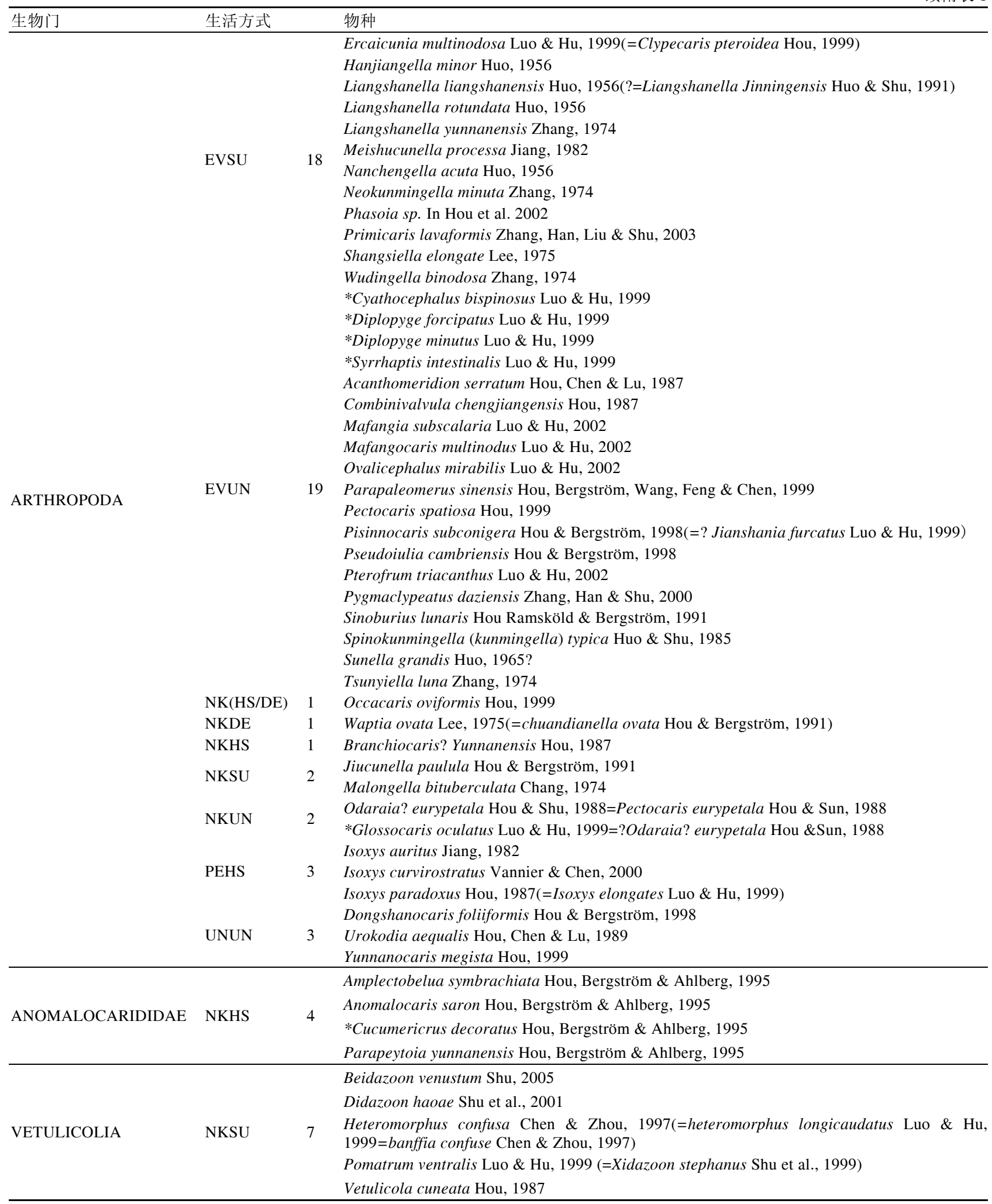


续附表 1

\begin{tabular}{|c|c|c|c|}
\hline 生物门 & 生活方式 & & 物种 \\
\hline \multirow{2}{*}{ VETULICOLIA } & \multirow{2}{*}{ NKSU } & & *Vetulicola rectangulata Luo \& Hu, 1999 \\
\hline & & & Yuyuanozoon magnificissimi Chen, Feng, Zhu, Ma \& Li, 2003 \\
\hline \multirow{2}{*}{ ECHINODERMS? } & \multirow{2}{*}{ ESSU? } & \multirow{2}{*}{2} & Dianchicystis jianshanensis Shu, Morris, Han, Zhang \& Liu, 2004 \\
\hline & & & Vetulocystis catenata Shu, Morris, Han, Zhang \& Liu, 2004 \\
\hline \multirow{10}{*}{ CHORDATA } & \multirow{2}{*}{ ESSU } & \multirow{2}{*}{2} & Shankouclava anningense Chen et al. 2003 \\
\hline & & & Cheungkongella ancestralis Shu et al., 2001 \\
\hline & \multirow{6}{*}{ NKSU } & \multirow{6}{*}{6} & Haikouella lanceolata Chen et al., 1999 (=Haikouella jianshanensis Shu et al., 2003) \\
\hline & & & Yunanozoon lividum Hou Ramsköld \& Bergström, 1991 \\
\hline & & & Zhongxiniscus intermedius Luo \& Hu, 2001 \\
\hline & & & Myllokunmingia fengjiaoa Shu et al, 1999 \\
\hline & & & *Haikouichthys ercaicunensis Shu et al, 1999 \\
\hline & & & *Zhongjianichthys rostradus Shu, 2003 \\
\hline & \multirow{2}{*}{ NKUN } & \multirow{2}{*}{2} & *Cathaymyrus diadexus Shu et al. 1996 \\
\hline & & & *Cathaymyrus haikouensis Luo \& Hu, 2001 \\
\hline \multirow{22}{*}{ UNKNOWN } & ESSU & 1 & Dinomischus isolatus Chen, Hou \& Lu, 1989 \\
\hline & \multirow{2}{*}{ ESSU? } & \multirow{2}{*}{2} & Cotyledion tylodes Luo \& Hu, 1999(=Cambrotentacus sanwuia Zhang et Shu, 2001) \\
\hline & & & Parvulonoda dubia Rigby \&Hou, 1995 \\
\hline & \multirow{6}{*}{ ESUN } & \multirow{6}{*}{6} & *Anthotrum robustus Luo \& Hu, 1999 \\
\hline & & & *Phacatrum tubifer Luo \& Hu, 1999 \\
\hline & & & Phasganula longa Luo \& Hu, 1999 \\
\hline & & & Jiucunia petalina Hou, Bergström, Wang, Feng \& Chen, 1999 \\
\hline & & & Phlogites longus Luo \& Hu, 1999(=Phlogites brevis Luo \& Hu, 1999) \\
\hline & & & Rhipitrus clavifer Luo \& Hu, 1999 \\
\hline & IVDE? & 1 & Maanshania crusticeps Hou, Bergström, Wang, Feng \& Chen, 1999 \\
\hline & IVSU & 1 & *Discoides abnormis Luo \& Hu, 1999 \\
\hline & IVUN & 1 & *Calathites spinalis Luo \& Hu, 1999 \\
\hline & PEHS & 1 & $\begin{array}{l}\text { Stellostomites eumorphus }(=\text { Eldonia eumorpha Sun \& Hou, 1987=Yunnanomedusa eleganta } \\
\text { Sun \& Hou, 1987) }\end{array}$ \\
\hline & \multirow[t]{4}{*}{ PEHS? } & \multirow[t]{4}{*}{1} & Rotadiscus grandis Sun \& Hou, 1987 \\
\hline & & & *Hippotrum spinatus Luo \& Hu, 1999 \\
\hline & & & *Macrocephalus elongates Luo \& Hu, 1999 \\
\hline & & & $\begin{array}{l}\text { *Oligonodus specialis Luo \& Hu, 1999(Chen, luo et al. } 2002 \text { 认为不是, 可能是某动物的部分 } \\
\text { 片断) }\end{array}$ \\
\hline & \multirow[t]{5}{*}{ UNUN } & \multirow[t]{5}{*}{8} & Pristioites bifarius Luo \& Hu, 1999 \\
\hline & & & Amiskwia sinica Luo \& $\mathrm{Hu}, 2002$ \\
\hline & & & Vetustovermis planus Glaessner, 1979(=Petalilium latus Luo \& Hu, 1999) \\
\hline & & & Malongitubus kuangshani $\mathrm{Hu}, 2005$ \\
\hline & & & Helcionella yunnanensis Zhang \& Babcock, 2002 \\
\hline \multirow{8}{*}{ COPROLITES } & & & Enteromophites intestinalis $\mathrm{Xu}, 2001$ \\
\hline & & & Longfengshania cordata Xu, 2002 \\
\hline & & & Paradelesseria sanguinea $\mathrm{Xu}, 2004$ \\
\hline & & & Vendotaenia cf. antiqua $\mathrm{Xu}, 2001$ \\
\hline & & & Fuxianospira gyrata Chen \& Zhou, 1997 \\
\hline & & & Megaspirellus houi Chen \& Erdtmann, 1991 \\
\hline & & & Plantulaformis sinensis $\mathrm{Xu}, 2002$ \\
\hline & & & Yuknessia sp. Chen \& Erdtmann, 1991 \\
\hline \multirow{3}{*}{ ALGAE } & & & Punctariopsis latifolia $\mathrm{Xu}, 2001$ \\
\hline & EAPP & 3 & Sinocylindra yunnanensis Chen \& Erdtmann, 1991 \\
\hline & & & Wahpia sp. $\mathrm{Hu}, 2005$ \\
\hline
\end{tabular}


附表 2 澄江生物群物种个体丰度表 ${ }^{a)}$

\begin{tabular}{|c|c|c|c|c|c|c|c|}
\hline & 物种(生物门) & 生活方式 & 个体数量 & & 物种(生物门) & 生活方式 & 个体数量 \\
\hline 1. & Kunmingella douvillei (Arth) & EVOM & 4838 & 58. & Luolishania sp. (Lobo) & EVHS & 12 \\
\hline 2. & Cricocosmia jinningensis (Pria) & IVHS & 2848 & 59. & Microdictyon sinicum (Lobo) & EVHS & 11 \\
\hline 3. & Diandongia pista (Brach) & ESSU & 2026 & 60. & Onychodictyon ferox (Lobo) & EVHS & 11 \\
\hline 4. & Leanchoilia illecebrosa (Arth) & EVHS & 899 & 61. & Vetulicola cuneata (Vetu) & NKSU & 10 \\
\hline 5. & Mafangscolex sinensis (Pria) & IVHS & 764 & 62. & Vetulicola sp. (Vetu) & NKSU & 10 \\
\hline 6. & Naraoia longicaudata (Arth) & EVHS & 573 & 63. & Xiangguangia sinica (Cnid) & ESHS & 10 \\
\hline 7. & $\begin{array}{l}\text { Archisaccophyllia kunmingensis } \\
\text { (Cnid) }\end{array}$ & ESSU & 494 & 64. & Kuamaia lata (Arth) & EVHS & 9 \\
\hline 8. & Naraoia spinosa (Arth) & EVHS & 485 & 65. & Palaeopriapulites parvus (Pria) & IVHS & 8 \\
\hline 9. & Chuandianella ovata (Arth) & EVSU & 399 & 66. & Cindarella eucalla (Arth) & EVHS & 8 \\
\hline 10. & Isoxys auritus (Arth) & PEHS & 365 & 67. & Retifacies abnormalis (Arth) & EVHS & 8 \\
\hline 11. & Wangyuia yunnanensis (Brach) & IVSU & 353 & 68. & Leptomitella conica (Pori) & ESSU & 8 \\
\hline 12. & Heliomedusa orienta (Brach) & ESSU & 341 & 69. & Independent elements.(Anom) & NKHS & 7 \\
\hline 13. & Ambrolinevitus ventricosus (Нyо) & $\mathrm{ES}(\mathrm{DE} / \mathrm{GR})$ & 270 & 70. & Combinivalvula chengjiangensis (Arth) & EVUN & 7 \\
\hline 14. & Sicyophorus rara (Pria) & IVDE & 262 & 71. & Urokidia aequalis (Arth) & EVUN & 7 \\
\hline 15. & Paraselkirkia sinica (Pria) & ISHS & 244 & 72. & Paucipoda haikouensis (Lobo) & EVHS & 7 \\
\hline 16. & Lingulella chengjiangensis (Brach) & IVSU & 233 & 73. & Burithes magnus (Un) & ESUN & 7 \\
\hline 17. & Kunmingella tipica (Arth) & EVOM & 230 & 74. & $\begin{array}{l}\text { Longtancunella chengjiangensis } \\
\text { (Brach) }\end{array}$ & ESSU & 7 \\
\hline 18. & New arthropod E & NKUN & 223 & 75 . & Maotianshania cylindrica (Pria) & IVHS & 6 \\
\hline 19. & Comptaluta leshanensis (Arth) & EVSU & 214 & 76. & Xandarella spectaculum (Arth) & EVHS & 6 \\
\hline 20. & Wutingaspis malungensis (Arth) & EVHS & 200 & 77. & Haikoucaris ercaiensis (Arth) & EVHS & 6 \\
\hline 21. & Wutingaspis tingi (Arth) & EVHS & 188 & 78. & Halichondrites sp. nov. (Pori) & ESSU & 6 \\
\hline 22. & Eoredlichia intermedia (Arth) & EVHS & 184 & 79. & Choia hindei (Pori) & ESSU & 5 \\
\hline 23. & Stellostomites eumorphus (Un) & PEHS & 132 & 80. & Hyalocinica sp (Pori) & ESSU & 5 \\
\hline 24. & Kunyangella cheni (Arth) & EVOM & 98 & 81. & Protospongia sp. (Pori) & ESSU & 5 \\
\hline 25. & Ambrolinevitus maximus (Нyо) & $\mathrm{ES}(\mathrm{DE} / \mathrm{GR})$ & 97 & 82. & Triticispongia diagonata (Pori) & ESSU & 4 \\
\hline 26. & Glossolites magnus (Нyо) & ESSU & 89 & 83. & Beidazoon venustum (Vetu) & NKSU & 3 \\
\hline 27. & Malungia laevigata (Arth) & EVDE & 86 & 84. & Brachiopoda indet. (Brach) & IVSU & 3 \\
\hline 28. & Arthropoda indet. & EVSU & 76 & 85. & Pseudoiulia cambriensis (Arth) & EVUN & 3 \\
\hline 29. & Haikouella lanceolata (Chor) & NKSU+ & 75 & 86. & Cambrohydra ercaia (Cnid) & EVHS & 3 \\
\hline 30. & Primicaris larvaformis (Arth) & EVSU & 75 & 87. & Linevitus opimus (Нyо) & $\mathrm{ES}(\mathrm{DE} / \mathrm{GR})$ & 3 \\
\hline 31. & Branchiocaris? Yunnanensis (Arth) & NKHS & 71 & 88. & Vetulicola retangulata (Vetu) & NKSU & 2 \\
\hline 32. & New lopobod B & EVHS & 61 & 89. & Pomatrum ventralis (Vetu) & NKSU & 2 \\
\hline 33. & $\begin{array}{l}\text { Amplectobelua symbrachiata } \\
\text { (Anom) }\end{array}$ & NKHS & 58 & 90. & Parapeytoia yunnanensi (Anom) & NKHS & 2 \\
\hline 34. & Archotuba conoidalis (Cnid) & ESSU & 54 & 91. & Pectocaris spatiosa (Arth) & EVUN & 2 \\
\hline 35. & Naraoia sp. (Arth) & EVHS & 49 & 92. & New arthropod C & EVHS & 2 \\
\hline 36. & Hazelia sp.nov (Pori) & ESSU & 42 & 93. & Phasganula longa (Un) & ESSU & 2 \\
\hline 37. & Sponge indet. (Pori) & ESSU & 34 & 94. & Ambrolinevitus platypluteus (Нyo) & $\mathrm{ES}(\mathrm{DE} / \mathrm{GR})$ & 2 \\
\hline 38. & Lingulellotreta malongensis (Brach) & IVSU & 33 & 95. & New arthropod D & NKUN & 1 \\
\hline 39. & Comptaluta inflata (Arth) & EVSU & 31 & 96. & Petalilium latus (Un) & NKUN & 1 \\
\hline 40. & Fuxianhuia protensa (Arth) & EVHS & 27 & 97. & Heteromorphus confusa (Vetu) & NKSU & 1 \\
\hline 41. & Isoxys sp. (Arth) & PEHS & 25 & 98. & Xiaoheiqingella peculiaris (Pria) & IVHS & 1 \\
\hline 42. & Saetaspongia densa (Pori) & ESSU & 25 & 99. & Worm A (Pria) & IVHS & 1 \\
\hline 43. & Bradorida indet. (Arth) & EVOM & 24 & 100. & Facivermis yunnanicus (Lobo) & IVHS & 1 \\
\hline 44. & Ercaicunia multinodosa (Arth) & EVSU & 23 & 101. & Acanthomeridion serratum (Arth) & EVUN & 1 \\
\hline 45. & Canadaspis laevigata (Arth) & EVHS & 23 & 102. & New arthropod A & EVUN & 1 \\
\hline 46. & Choiaella radiata (Pori) & ESSU & 23 & 103. & New arthropod B & EVUN & 1 \\
\hline 47. & Paraleptomitella dictyodroma (Pori) & ESSU & 23 & 104. & Kuanyangia pustulosa (Arth) & EVOM & 1 \\
\hline 48. & Leptomitus teretiusculus (Pori) & ESSU & 22 & 105 . & New lobopod A & EVHS & 1 \\
\hline 49. & Yunnanocephalus yunnanensis (Arth) & EVHS & 21 & 106. & Hallucigenia fortis (Lobo) & EVHS & 1 \\
\hline 50. & Corynetis brevis (Pria) & IVHS & 20 & 107. & Wudingella binodosa (Arth) & EVHS & 1 \\
\hline 51. & Isoxys paradoxus (Arth) & PEHS & 19 & 108. & Chengjiangocaris longiformis (Arth) & EVHS & 1 \\
\hline 52. & Archotuba conoidalis (Cnid) & ESSU & 19 & 109. & Jianfengia multisegmentalis (Arth) & EVHS & 1 \\
\hline 53. & Anomalocaris saron (Anom) & NKHS & 17 & 110. & Squamacula clypeata (Arth) & EVHS & 1 \\
\hline 54. & Isoxys curvirostratus (Arth) & PEHS & 16 & 111. & Cotyledion tylodes $(\mathrm{Un})$ & ESUN & 1 \\
\hline 55. & Cardiodictyon catenulum (Lobo) & EVHS & 16 & 112. & Paraleptomitella globula (Pori) & ESSU & 1 \\
\hline 56. & Allonia phrixothrix (Chan) & ESSU & 16 & 113. & New chancelloriids A & ESSU & 1 \\
\hline 57. & Sponge gemmule (Pori) & ESSU & 14 & 114. & Dinomischus venustus (Un) & ESSU & 1 \\
\hline
\end{tabular}

a) Anom, Anomalocarididae; Arth, Arthropoda; Brach, Brachiopoda; Chan, Chancelloriids; Chor, Chordata; Cnid, Cnidaria; Hyo, Hyolitha; Lobo, Lobopodia; Pori, Porifera; Pria, Priapulida; Un, Unknown; Vetu, Vetulicolia 